\title{
Isotopic variation of parity violation in atomic ytterbium: method of measurements and analysis of systematic effects
}

\author{
D. Antypas* \\ Helmholtz-Institut Mainz, Mainz 55128, Germany \\ A.M. Fabricant \\ Johannes Gutenberg-Universität Mainz, Mainz 55128, Germany \\ J.E. Stalnaker \\ Department of Physics and Astronomy, Oberlin College, Oberlin, Ohio 44074, USA \\ K. Tsigutkin \\ $A S M L$, Veldhoven, The Netherlands \\ V.V. Flambaum \\ School of Physics, University of New South Wales, Sydney 2052, Australia and \\ Johannes Gutenberg-Universität Mainz, Mainz 55128, Germany \\ D. Budker \\ Johannes Gutenberg-Universität Mainz, Mainz 55128, Germany \\ Helmholtz-Institut Mainz, Mainz 55128, Germany and \\ Department of Physics, University of California at Berkeley, California 94720-300, USA
}

(Dated: May 20, 2019)

\begin{abstract}
We present a detailed description of experimental studies of the parity violation effect in an isotopic chain of atomic ytterbium $(\mathrm{Yb})$, whose results were reported in a recent Letter [Antypas et al., Nat. Phys. 15, 120 (2019) 1]]. We discuss the principle of these measurements, made on the $\mathrm{Yb} 6 \mathrm{~s}^{2}{ }^{1} \mathrm{~S}_{0} \rightarrow 5 \mathrm{~d} 6 \mathrm{~s}^{3} \mathrm{D}_{1}$ optical transition at $408 \mathrm{~nm}$, describe the experimental apparatus, and give a detailed account of our studies of systematic effects in the experiment. Our results offer the first direct observation of the isotopic variation in the atomic parity violation effect, a variation which is in agreement with the prediction of the Standard Model. These measurements are used to constrain electron-proton and electron-neutron interactions, mediated by a light $Z^{\prime}$ boson.
\end{abstract}

PACS numbers: 11.30.Er, 32.90.a+

\section{INTRODUCTION}

The investigation of weak-force-induced effects in atomic systems has been the focus of experiments in the last four decades (see, for example, reviews [2 4]). The first experiments were motivated by the work of Bouchiat and Bouchiat [5] which showed that weak-interactioninduced observables in atoms are enhanced and therefore are detectable in systems with large atomic number. This finding followed the earlier recognition by Zel'dovich 6 that the electron-nucleus weak interaction induces optical rotation in atomic media. Atomic physics techniques have been employed to study the parity violation (PV) at low energy. Combined with atomic structure calculations, these efforts have determined the nuclear weak charge, a quantity predicted in the Standard Model (SM), thereby testing the SM. Such tabletop experiments are complementary to studying the electroweak sector of the $\mathrm{SM}$ at high energies.

\footnotetext{
* dantypas@uni-mainz.de
}

The first observations of atomic PV were made in bismouth (Bi) 7], thallium (Th) 8] and cesium (Cs) 9]. Accurate determinations of the PV effects were made in Bi [10, lead $(\mathrm{Pb})$ [1, 12, Th [13, 14] and Cs 15, 16. The highest measurement accuracy was achieved in Cs [15. Combined with precise atomic-structure calculations [17, the Cs experiment resulted in a determination of the nuclear weak charge at the level of $0.5 \%$. This result is the most-precise-to-date low-energy test of the SM.

Atomic PV experiments can additionally be platforms to study nuclear physics as well as physics beyond the SM. Measurements of nuclear-spin-dependent contributions to the PV effect probe the nuclear anapole moment 18 20, which has only been observed to date in the Cs experiment 15]. Determining an anapole provides information about the so-far poorly understood weak meson-nucleon couplings that characterize the hadronic weak interactions, as formulated in the model of Desplanques, Donoghue, and Hollstein [21. Measurements of PV across a chain of isotopes of the same element, first proposed in 22, have the potential probe to physics beyond the SM [23, 24, such as to search for extra 
light bosons that mediate parity-violating interactions between the electron and nucleons 25. The isotopic comparison method can be also employed to probe the variation of the neutron distribution in the nucleus, and to test nuclear models [24, 26].

A number of PV experiments are currently underway, that make use of neutral atoms, as well as atomic ions and molecules. Of these, an experiment in Fr [27] is aiming to determine the nuclear weak charge, as well as to measure the anapole moment of Fr nuclei. Another project with Fr, currently at a preliminary stage [28], also aims to measure the weak charge and anapole. An experiment using a single trapped $\mathrm{Ra}^{+}$ion 29, aims to determine the nuclear weak charge in several different isotopes. An ongoing experiment in Cs 30, is primarily focused on a cross-check measurement of the Cs anapole moment. Improved measurements of PV are underway in Dy 31, in which a previous experiment yielded an effect consistent with zero [32. Finally, an effort with BaF [33, 34] has recently demonstrated adequate sensitivity to make an accurate determination of the anapole moment of the $\mathrm{Ba}$ nucleus.

Accurate extraction of the nuclear weak charge from PV measurements requires atomic calculations of adequate precision. Such a precision can be reached in simple atomic systems such as Cs, Fr or $\mathrm{Ra}^{+}$(the Cs theory, for example, is at the $0.5 \%$ level of uncertainty [17]), thus making it possible for a single-isotope measurement to be a probe of the SM. In Yb, which has two valence electrons, existing atomic calculations have a relatively large uncertainty at the $10 \%$ level 35, 36. Significant advancement in the $\mathrm{Yb}$ theory is required to enable a competitive determination of the $\mathrm{Yb}$ weak charge. With regard to searching for physics beyond the SM via atomic $\mathrm{PV}$, the merit of using Yb lies in the availability of a number of stable isotopes, that makes it possible to employ the isotopic comparison method [22]. The same method could also be used to probe the neutron distributions of the $\mathrm{Yb}$ nuclei.

We recently reported on measurements of $\mathrm{PV}$ in the $6 \mathrm{~s}^{2}$ ${ }^{1} \mathrm{~S}_{0} \rightarrow 5 \mathrm{~d} 6 \mathrm{~s}{ }^{3} \mathrm{D}_{1}$ optical transition at $408 \mathrm{~nm}$ in a chain of four nuclear-spin-zero $\mathrm{Yb}$ isotopes [1]. That work provided an observation of the isotopic variation of the $\mathrm{PV}$ effect, and was part of a program that focuses on nuclear spin-dependent PV, neutron skins, as well as on searching for light bosons beyond SM. These results built upon an earlier observation of the $\mathrm{Yb} \mathrm{PV}$ effect [37, 38. The previous measurement confirmed the large size of the effect, which was first estimated in [39], with more elaborate calculations following up [35, 36, 40]. Here we present in detail the method utilized for these isotopic-chain measurements, discuss the experimental apparatus, and provide an analysis of systematic effects.

\section{EXPERIMENTAL METHOD}

To study $\mathrm{PV}$ in $\mathrm{Yb}$, we make use of the $6 \mathrm{~s}^{2}{ }^{1} \mathrm{~S}_{0} \rightarrow$ $5 \mathrm{~d} 6 \mathrm{~s}{ }^{3} \mathrm{D}_{1}$ optical transition at $408 \mathrm{~nm}$ (fig. 1). The experimental principle was described in 38. A small electric-dipole $(E 1)$ transition amplitude arises between the ${ }^{1} \mathrm{~S}_{0}$ and ${ }^{3} \mathrm{D}_{1}$ states, mainly due to weak-interactioninduced mixing between the ${ }^{3} \mathrm{D}_{1}$ and ${ }^{1} \mathrm{P}_{1}$ states. The application of a quasi-static electric field creates additional (Stark) mixing between the same states [41, and introduces a Stark-induced $E 1$ amplitude for the $408 \mathrm{~nm}$ transition. A static magnetic field is also applied to the atoms to split the Zeeman sublevels of the excited ${ }^{3} \mathrm{D}_{1}$ state. With appropriate choice of geometry for the applied static and optical fields, the Stark and PV amplitudes interfere [42]. The sign of this interference in the $408 \mathrm{~nm}$ excitation rate can be changed by making field reversals, allowing extraction of the P-odd part of this rate from the larger P-even background. For the geometry of fields in the present experiment (fig. 2), the Stark-PV interference is proportional to the following pseudo-scalar rotational invariant [42, 43]:

$$
(\overrightarrow{\mathcal{E}} \cdot \vec{B}) \cdot([\vec{E} \times \overrightarrow{\mathcal{E}}] \cdot \vec{B}),
$$

where $\vec{E}, \overrightarrow{\mathcal{E}}$ and $\vec{B}$ are, respectively, the quasi-static electric, optical and magnetic fields applied to the atoms. The Stark and PV amplitudes for the $m=0 \rightarrow m^{\prime}$ component of the ${ }^{1} \mathrm{~S}_{0} \rightarrow{ }^{3} \mathrm{D}_{1}$ transition are given by [38]:

$$
\begin{gathered}
A_{m^{\prime}}^{\text {Stark }}=i \beta(-1)^{m^{\prime}}(\vec{E} \times \overrightarrow{\mathcal{E}})_{-m^{\prime}}, \\
A_{m^{\prime}}^{P V}=i \zeta(-1)^{m^{\prime} \overrightarrow{\mathcal{E}}_{-m^{\prime}}}
\end{gathered}
$$

where $\beta=2.24(12) \cdot 10^{-8} e a_{0} /(\mathrm{V} / \mathrm{cm})$ is the vector polarizability of the transition, determined in [44, 45], and $\zeta$ is the $E 1$ transition moment arising primarily from the $\mathrm{PV}$-mixing of the ${ }^{3} \mathrm{D}_{1}$ and ${ }^{1} \mathrm{P}_{1}$ states. The parameter $\zeta$ is proportional to the nuclear weak charge. The element $V_{q}$ is the $q$-component of the vector $\vec{V}$ in the spherical basis. The results presented here come from measurements on the $m=0 \rightarrow m^{\prime}=0$ transition component, whereas the previous experiment [37, 38] utilized all three magnetic sublevels of the $408 \mathrm{~nm}$ transition to determine the PV-effect.

The effects of a magnetic-dipole $(M 1)$ transition between the ${ }^{1} \mathrm{~S}_{0}$ and ${ }^{3} \mathrm{D}_{1}$ states, whose amplitude is $\approx 930$ times greater than that of the PV amplitude, are suppressed in this experiment. The primary method of suppression is the appropriate choice of the geometry of fields in the interaction region. This geometry is chosen such that the Stark and PV amplitudes are in phase and therefore allowed to interfere, but the $M 1$ and Stark amplitudes are nominally out of phase and do not interfere. As a result, the $M 1$-related systematic contributions to the PV measurements are practically eliminated. Additional suppression of $M 1$-systematics occurs because the 


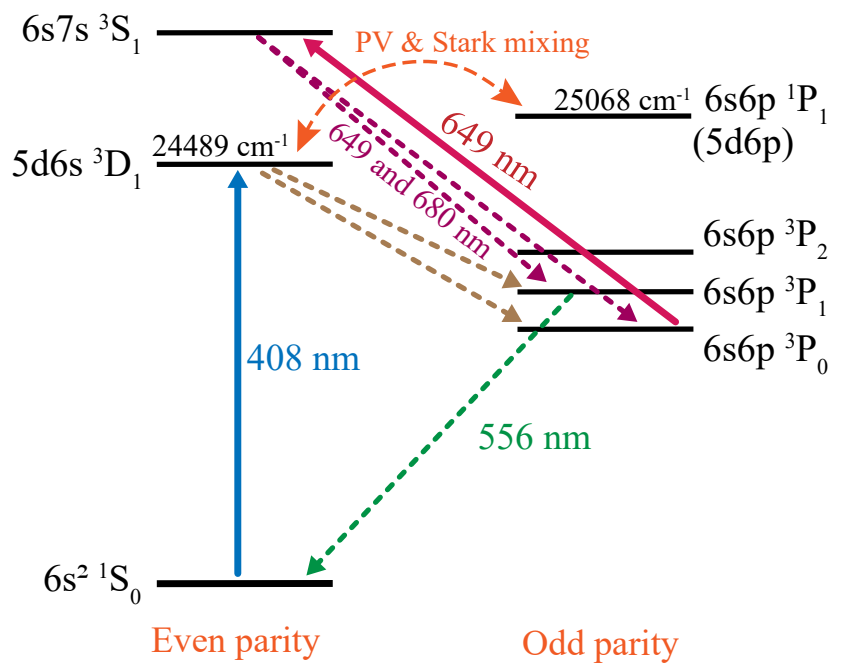

FIG. 1. (Color online) Partial energy level diagram of $\mathrm{Yb}$ with levels related to the PV experiment. Solid straight lines indicate excitations and dashed straight lines indicate decays. The PV effect arises primarily due to weak-interactioninduced mixing of the ${ }^{3} \mathrm{D}_{1}$ and ${ }^{1} \mathrm{P}_{1}$ levels. About $67 \%$ of atoms excited to the ${ }^{3} \mathrm{D}_{1}$ level decay to the metastable ${ }^{3} \mathrm{P}_{0}$ level. These atoms are detected by subsequent excitation to ${ }^{3} \mathrm{~S}_{1}$ and collection of fluorescence from decays at 556, 649 and $680 \mathrm{~nm}$.

${ }^{1} \mathrm{~S}_{0} \rightarrow{ }^{3} \mathrm{D}_{1}$ excitation is done with a standing-wave optical field. Analysis of the residual contribution of the $M 1$ transition to the present measurements is carried out in Appendix A.

In the absence of non-reversing fields and field misalignments, the magnetic field is along the z-axis, $\vec{B}=$ $B_{z} \hat{z}$, and the electric field along the x-axis, $\vec{E}=\left(E_{d c}+\right.$ $\left.E_{0} \cos \omega t\right) \hat{x}$. This field consists of a component oscillating at frequency $\omega(\omega / 2 \pi=19.9 \mathrm{~Hz})$ as well as a dc-term. The ac-component, of typical amplitude $1.2 \mathrm{kV} / \mathrm{cm}$, is primarily responsible for the required Stark-induced mixing between ${ }^{3} \mathrm{D}_{1}$ and ${ }^{1} \mathrm{P}_{1}$ states. The change of the ac-field direction is the primary parity reversal in the experiment. The dc term $E_{d c}(\approx 6 \mathrm{~V} / \mathrm{cm})$ is used to optimize detection conditions for the Stark-PV interference. The optical field is linearly polarized and propagates along $\mathrm{x}$ : $\overrightarrow{\mathcal{E}}=\mathcal{E}(\sin \theta \hat{y}+\cos \theta \hat{z})$. Under these conditions, the excitation rate for the $m=0 \rightarrow m^{\prime}$ transition component has the form:

$R_{m^{\prime}} \propto\left|A_{m^{\prime}}^{\text {Stark }}+A_{m^{\prime}}^{P V}\right|^{2}=R_{m^{\prime}}^{[0]}+R_{m^{\prime}}^{[1]} \cos \omega t+R_{m^{\prime}}^{[2]} \cos 2 \omega t$

This rate consists of a dc term of amplitude $R_{m^{\prime}}^{[0]}$ and components oscillating at frequencies $\omega$ and $2 \omega$ with respective amplitudes $R_{m^{\prime}}^{[1]}$ and $R_{m^{\prime}}^{[2]}$. For the $0 \rightarrow 0$ transition these terms are as follows:

$$
\begin{aligned}
R_{0}^{[0]}=2 \mathcal{E}^{2} \beta^{2} E_{0}^{2} \sin ^{2} \theta+ & 4 \mathcal{E}^{2} \beta^{2} E_{d c}^{2} \sin ^{2} \theta \\
& +8 \mathcal{E}^{2} \beta E_{d c} \zeta \cos \theta \sin \theta,
\end{aligned}
$$

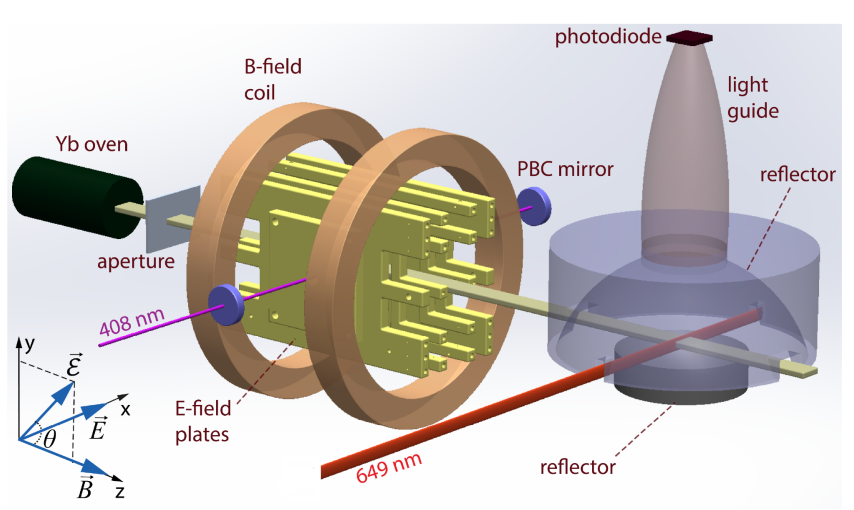

FIG. 2. (Color online) Schematic of the Yb atomic-beam apparatus. The $\mathrm{Yb}$ atoms effuse from the oven into the interaction region, where they are excited by $408 \mathrm{~nm}$ light in the presence of applied electric and magnetic fields. The atoms that are excited are detected in the downstream detection region via excitation from the metastable state at $649 \mathrm{~nm}$. Figure reproduced from [1].

$$
\begin{gathered}
R_{0}^{[1]}=8 \mathcal{E}^{2} \beta E_{0} \zeta \cos \theta \sin \theta+8 \mathcal{E}^{2} \beta^{2} E_{0} E_{d c} \sin ^{2} \theta \\
R_{0}^{[2]}=2 \mathcal{E}^{2} \beta^{2} E_{0}^{2} \sin ^{2} \theta .
\end{gathered}
$$

Only terms independent of or linear in the weakinteraction parameter $\zeta$ are retained in (4), (6) and (7). Phase-sensitive detection at the frequencies $\omega$ and $2 \omega$ provides the amplitudes $R_{0}^{[1]}$ and $R_{0}^{[2]}$. Their ratio is related to the ratio of the PV-and Stark-induced transition moments:

$$
r_{0} \equiv \frac{R_{0}^{[1]}}{R_{0}^{[2]}}=\frac{4 E_{d c}}{E_{0}}+\frac{4 \zeta}{\beta E_{0}} \cot \theta .
$$

Observation of the change in $r_{0}$ under the second parity reversal, i.e. a $\pm \pi / 2$ rotation of the light polarization plane, yields the ratio $\zeta / \beta$. In addition to the $E$ - and $\theta$ - reversals (parity reversals), the magnetic field $\vec{B}$ as well as the polarity of $E_{d c}$ are also reversed, in order to study and minimize systematic contributions, not explicitly shown in (8).

Misalignments of the applied fields, non-reversing field components, as well as imperfections in the optical polarization alter the ideal situation discussed above, and result in additional contributions to the transition rate (4) and to the ratio (8). The applied electric field and magnetic fields are most generally given by:

$$
\vec{E}=\left(E_{d c}+E_{0} \cos \omega t\right) \hat{x}+\left(e_{y}+e_{y}^{r} \cos \omega t\right) \hat{y}+\left(e_{z}+e_{z}^{r} \cos \omega t\right) \hat{z},
$$

$$
\vec{B}=\left(b_{x}+f_{B} b_{x}^{r}\right) \hat{x}+\left(b_{y}+f_{B} b_{y}^{r}\right) \hat{y}+\left(b_{z}+f_{B} B_{z}\right) \hat{z} .
$$

The component $v_{i}$ denotes the stray (non-reversing) component of the vector $\vec{V}$ along the i-axis, and $v_{i}^{r}$ the reversing $\vec{V}$ component along the same axis. A B-field 
flip parameter $f_{B}= \pm 1$ is introduced in 10 . All the field components containing the term $f_{B}$ reverse with the main magnetic field. Allowing for an ellipticity in the nominally linearly polarized optical field, $\overrightarrow{\mathcal{E}}$ becomes:

$$
\overrightarrow{\mathcal{E}}=\mathcal{E}\left(\sin \theta \hat{y}+\cos \theta e^{i \phi} \hat{z}\right)
$$

As discussed in 38, a rotation operation has to be applied to the fields of $(9),(10)$ and $\sqrt{11}$ so that the rotated $\vec{B}$ is along $\mathrm{z}$. The transition rate (4), as well as the harmonics amplitudes $R_{0}^{[1]}$ and $R_{0}^{[2]}$, acquire then a large number of terms. A series expansion in the field imperfections and $\zeta$ yields a harmonics ratio $r_{0}\left(\theta, f_{B}\right)$, in which, in addition to the $\mathrm{PV}$-related term $\zeta / \beta$, terms that transform in the same way as $\zeta / \beta$ under the $\theta$-reversal, are also present. The full expression for $r_{0}\left(\theta, f_{B}\right)$ in the presence of apparatus imperfections is given in Appendix A. A simplified expression, that includes the only significantly contributing $\mathrm{PV}$-mimicking term, is the following:

$r_{0}\left(\theta, f_{B}\right)=\frac{4 E_{d c}}{E_{0}}+\left[\frac{4 \zeta}{\beta E_{0}}+\frac{4\left(b_{x}+f_{B} b_{x}^{r}\right) e_{y}}{f_{B} B_{z} E_{0}}\right] \cot \theta \cos \phi$

There are four different values of $r_{0}\left(\theta, f_{B}\right)$, corresponding to the two possible values of the polarization angle $(\theta \approx \pm \pi / 4)$ and magnetic field direction $\left(f_{B}= \pm 1\right)$, and four different ways to combine these values. These combinations, labeled $K_{i}(\mathrm{i}=1,2,3,4)$, are computed using the full expression for $r_{0}\left(\theta, f_{B}\right)$ (see Appendix A) as follows:

$$
\left(\begin{array}{l}
K_{1} \\
K_{2} \\
K_{3} \\
K_{4}
\end{array}\right)=\left(\begin{array}{llll}
+1 & -1 & +1 & -1 \\
-1 & -1 & +1 & +1 \\
-1 & +1 & +1 & -1 \\
+1 & +1 & +1 & +1
\end{array}\right) \cdot\left(\begin{array}{l}
r_{0}\left(\theta_{+},+1\right) \\
r_{0}\left(\theta_{-},+1\right) \\
r_{0}\left(\theta_{+},-1\right) \\
r_{0}\left(\theta_{-},-1\right)
\end{array}\right)
$$

The values of $K_{i}$ are given in Table I. One of these $\left(K_{1}\right)$ yields the ratio $\zeta / \beta$; the others provide important information about parasitic fields and overall measurement consistency. Some of the $K_{i}$ values are expressed in terms of the polarization parameter $p$, defined as:

$$
p=\cot \theta_{+} \cos \phi_{+}-\cot \theta_{-} \cos \phi_{-},
$$

with $p \approx 2$ in the experiment. The angles $\phi_{ \pm}$are the ellipticity-related parameters corresponding to the angles $\theta_{ \pm}$. Examination of the terms in $K_{1}$ shows that a precision determination of $\zeta / \beta$ requires, aside from accurate knowledge of $E_{0}$, a measurement of the false-PV contribution $e_{y} b_{x}^{r} / B_{z}$ as well as a measurement of the parameter $p$. Methods to make these measurements are discussed in section IV.

\section{APPARATUS}

The PV isotopic comparison experiment was carried out with a newly built atomic-beam apparatus which has
TABLE I. The four combinations of harmonics ratio $r_{0}\left(\theta, f_{B}\right)$ values, corresponding to the two orientations of the polarization angle $\left(\theta_{ \pm} \approx \pm \pi / 4\right)$ and magnetic field $\left(f_{B}= \pm 1\right)$. The angles $\phi_{ \pm}$are the small optical field ellipticity-related parameters for the polarization states with angles $\theta_{ \pm}$, respectively.

\begin{tabular}{cc}
\hline \hline Combination & $\left(\frac{8 \zeta}{\beta E_{0}}+\frac{8 b_{x}^{r} e_{y}}{B_{z} E_{0}}\right) p$ \\
$K_{1}$ & $\frac{16 b_{x} e_{z}}{B_{z} E_{0}}-\frac{32 b_{y} \zeta}{\beta B_{z} E_{0}}$ \\
$K_{2}$ & $-\frac{8 b_{x} e_{y}}{\beta B_{z} E_{0}} p$ \\
$K_{3}$ & $\frac{16 E_{d c}}{E_{0}}-\frac{16 b_{x}^{r} e_{z}}{B_{z} E_{0}}+\frac{32 b_{y} \zeta}{\beta B_{z} E_{0}}$ \\
$K_{4}$ & \\
\hline \hline
\end{tabular}

increased statistical sensitivity and better ability to study and control systematics, compared to that of [37, 38].

A schematic of the in-vacuum setup is shown in fig. 2. An $\mathrm{Yb}$ atomic beam is produced with an oven heated to $\approx 550{ }^{\circ} \mathrm{C}$. Atoms exiting the oven nozzle travel a distance of $\approx 28 \mathrm{~cm}$ to reach the interaction region, with a mean longitudinal velocity of $\approx 290 \mathrm{~m} / \mathrm{sec}$ and a transverse velocity spread of $\approx 8 \mathrm{~m} / \mathrm{s}$ (Full Width at Half Maximum-FWHM). In the interaction region, the atoms intercept the $408 \mathrm{~nm}$ standing-wave optical field, tuned to excite the ${ }^{1} S_{0} \rightarrow{ }^{3} D_{1}$ transition. This light circulates in a power-build-up cavity (PBC), which has a finesse of $\approx 550$ and is used to enhance the light power available to excite atoms, but also to suppress the effects of the $M 1-$ Stark interference. The circulating power is measured by recording the light transmitted through the $\mathrm{PBC}$, and it is actively stabilized, to a level of $\approx 55 \mathrm{~W}$. This stabilization results in negligible contribution of intracavity power noise to noise in detection of the excitation rate on the $408 \mathrm{~nm}$ transition. The waist $\left(1 / e^{2}\right.$ intensity radius) of the optical beam in the interaction region is $w_{0} \approx 310 \mu \mathrm{m}$, corresponding to an intensity of $\approx 18$ $\mathrm{kW} / \mathrm{cm}^{2}$, or to an optical field applied to the atoms of amplitude $\approx 3.7 \mathrm{kV} / \mathrm{cm}$. This amplitude is about three times greater than the typical amplitude of the quasi-dc field applied in the interaction region $E_{0} \approx 1.2 \mathrm{kV} / \mathrm{cm}$. The intracavity power level is a compromise between the need for large $408 \mathrm{~nm}$ excitation rate and unwanted distortion and broadening in the transition lineshape, which appears for an intracavity intensity around $10 \mathrm{~kW} / \mathrm{cm}^{2}$ and becomes excessive for intensities above the current level of $18 \mathrm{~kW} / \mathrm{cm}^{2}$. This distortion has been studied extensively in 45, 46] and can be removed, if needed, using methods reported in [47. It arises in the presence of an off-resonant ac-Stark effect, induced by the intense standing-wave field. Owing to the imperfect collimation of the atomic beam, most atoms traversing the standingwave fly through many nodes and anti-nodes of the field, and in the presence of the ac-Stark effect, experience amplitude, and effectively frequency modulation (the latter 
occurs due to ac-Stark-induced modulation of the energy levels). This combined amplitude and frequency modulation results in a complex lineshape for the $408 \mathrm{~nm}$ transition, that is shown in fig. 3 .

The required electric field is applied to the atoms with a system of gold-coated electrodes. This system consists of two main plates, approximately $10 \times 10 \mathrm{~cm}^{2}$, spaced by $5.5045(20) \mathrm{cm}$. A set of eight surrounding electrodes is employed to increase field uniformity as well as to apply auxiliary field components in either the y- or z- direction, for systematics studies. Six high-voltage amplifiers and a system of voltage dividers are used to bias the main plates and surrounding electrodes. Simulations of the electric field with COMSOL ${ }^{\mathrm{R}}$ yield a value for the primary field of $\left[1-2.7(3) \cdot 10^{-4}\right] \cdot V / d$, where $V$ is the potential difference between the plates, and $d$ is the plate spacing. The non-uniformity of the field within the 1.5 $\mathrm{cm}$ wide interaction region (whose diameter is $0.6 \mathrm{~mm}$ ) is lower than $0.1 \%$. The magnetic field in the interaction region of $93 \mathrm{G}$ is applied with a pair of round in-vacuum coils, which have nearly Helmholtz geometry. Additional sets of coils are used to cancel the residual field in the interaction region (to within $20 \mathrm{mG}$ ), as well as to apply additional field components for studies and control of systematics.

Detection of the $408 \mathrm{~nm}$ excitations in the interaction region is done downstream in the path of the atoms using an efficient detection scheme described in detail in [38, 48. The fraction of atoms $(\approx 65 \%)$ that decayed to the ${ }^{3} \mathrm{P}_{0}$ metastable state after undergoing the 408 $\mathrm{nm}$ transition, are further excited with $\approx 120 \mu \mathrm{W}$ of 649 $\mathrm{nm}$ light to the ${ }^{3} \mathrm{~S}_{1}$ state (see fig. 1), in the region of an optimized light collector (fig. 2). The light collector directs the induced fluorescence at 556, 649 and 680 $\mathrm{nm}$ to a light-pipe which guides light out of the vacuum chamber and onto the surface of a large-area photodiode, whose photocurrent is amplified with a low-noise transimpedance amplifier. This amplifier has a 1 G $\Omega$ transimpedance and $\approx 1.1 \mathrm{kHz}$ bandwidth. The overall detection efficiency of the $408 \mathrm{~nm}$ transitions is an estimated $25 \%$ [49].

The $408 \mathrm{~nm}$ laser system is a frequency-doubled Ti:Sapphire laser $\left(\mathrm{M}^{2}\right.$ SolStiS+ECD-X) outputting $\approx 1$ $\mathrm{W}$ of near-UV light. The laser frequency is stabilized to an internal reference cavity, with a resulting linewidth of less than $100 \mathrm{kHz}$. The short-term stability of the system is sufficiently good so that we use the internal cavity as the short-term frequency reference. The PBC is stabilized to this reference through frequency-modulation spectroscopy; the PBC length is modulated at $29 \mathrm{kHz}$ using a piezo-transducer onto which one of the cavity mirrors is mounted, and the demodulated PBC transmission is applied back to the piezo with an electronic filter. During an experiment, the laser frequency is locked to the peak of the resonance profile of the atomic transition (see fig. 3). For this, the Ti:Sapphire frequency is modulated at $138 \mathrm{~Hz}$ (with an amplitude of $\approx 200 \mathrm{kHz}$ ) and the recorded detection-region fluorescence is demod-

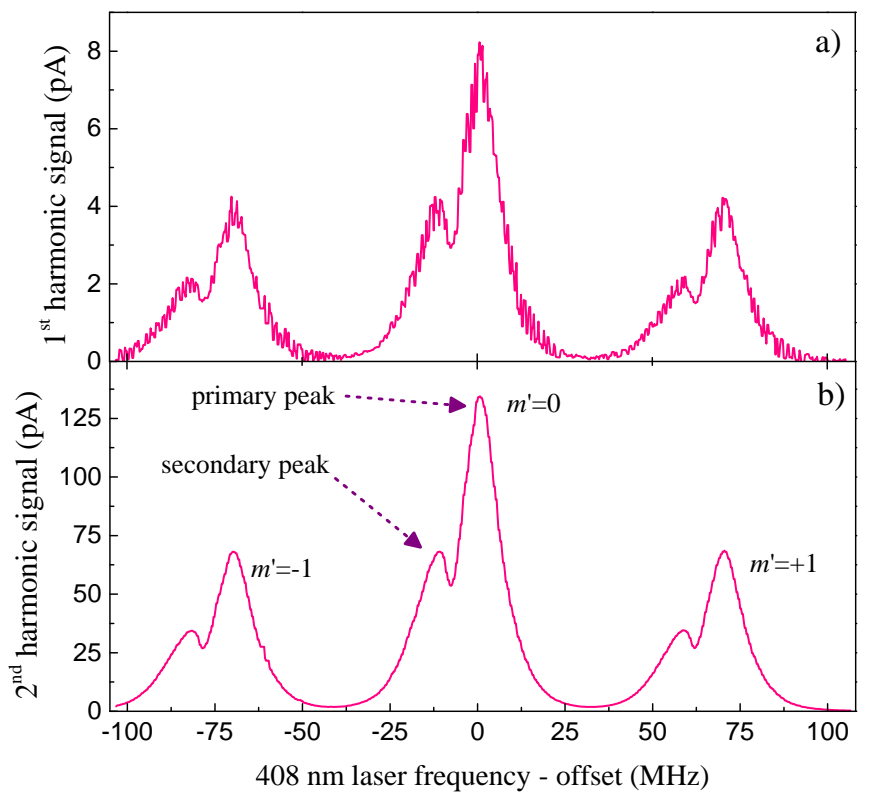

FIG. 3. (Color online) Spectral profile of the ${ }^{174} \mathrm{Yb}^{1} \mathrm{~S}_{0} \rightarrow{ }^{3} \mathrm{D}_{1}$ transition. a): $1^{\text {st }}$ harmonic present in the excitation rate $\mathrm{b}$ ): $2^{n d}$ harmonic. These spectra are obtained by scanning the $408 \mathrm{~nm}$ laser frequency around the center of the resonance and measuring the respective harmonic contributions to the photocurrent from the detection region (see text). The three Zeeman components of the transition ${ }^{1} \mathrm{~S}_{0}, m=0 \rightarrow{ }^{3} \mathrm{D}_{1}$, $m^{\prime}=0, \pm 1$ are fully resolved in the presence of $\mathrm{a} \approx 93 \mathrm{G}$ magnetic field in the interaction region. The applied electric field has an ac-amplitude of $E_{0}=1000 \mathrm{~V} / \mathrm{cm}$ and a dc-component of $E_{d c} \approx 14.54 \mathrm{~V} / \mathrm{cm}$. The low optical intensity peak is 18 $\mathrm{MHz}$ wide (FWHM), and is distorted at higher intensity due to the effects of the off-resonant ac-Stark effect in the presence of the standing-wave field circulating in the PBC (see text). Figure adapted from supplementary material of [1].

ulated with a lock-in amplifier, whose output is fed back to the laser, through an electronic filter of low $(\approx 1 \mathrm{~Hz})$ bandwidth. This scheme ensures long-term frequency stability for the $408 \mathrm{~nm}$ laser system.

The $649 \mathrm{~nm}$ laser system, whose output is used to excite the $60 \mathrm{MHz}$ wide ${ }^{3} \mathrm{P}_{0} \rightarrow{ }^{3} \mathrm{~S}_{1}$ detection transition, is an external-cavity diode laser (Vitawave ECDL-6515R). To suppress frequency noise of this laser, its frequency is locked to the side-of-fringe of an airtight Fabry-Perot (FP) resonator. The resonator length is in turn stabilized with slow feedback to a set laser frequency, whose reading is made with a wavemeter (HighFinesse WSU2). This double-stage scheme ensures short- and long-term stability so that the impact of frequency excursions of the laser on the detection of the $408 \mathrm{~nm}$ transition is negligible.

Precise polarization control of the intracavity optical field, as well as continuous measurement of the PBC polarization, are needed in the experiment. The linear polarization of the light coupled to the PBC is set with a half-wave plate mounted on a motorized rotation stage. This polarization is measured with a balanced polarime- 
ter, placed at the output of the PBC. The polarimeter makes use of a Glan-Taylor polarizer that analyzes a small fraction of the light transmitted through the PBC. This light is picked off with a wedge window placed at near-normal incidence in the path of the beam exiting the PBC. The two orthogonal polarization states at the output of the polarizer are measured with a pair of amplified photodetectors. The polarizer axis is set so that the polarimeter is nominally balanced for the $\theta_{ \pm}$polarization angles. The small polarization ellipticity in the PBC, whose value is also required for an accurate PV-effect measurement, is determined using a scheme outlined in section IVA2,

Lock-in amplifiers are used to measure the $1^{\text {st }}$ and $2^{\text {nd }}$ harmonics present in the $408 \mathrm{~nm}$ excitation rate (models Signal Recovery SR7265 and Zurich Instruments MLFI, respectively). For typical electric field amplitude $E_{0} \approx 1$ $\mathrm{kV} / \mathrm{cm}$, the contribution to the ratio $r_{0}$ (12) from the $\mathrm{PV}$ effect is $4 \zeta / \beta E_{0} \approx 10^{-4}$. Due to the small size of the $1^{\text {st }}$ harmonic $R_{0}^{[1]}$, its detection in the presence of a much larger $2^{\text {nd }}$ harmonic amplitude $R_{0}^{[2]}$ is technically challenging. Two steps are taken to circumvent this issue. First, a field $E_{d c} \approx 6.3 \mathrm{~V} / \mathrm{cm}$ is applied in the interaction region. The resulting contribution $4 E_{d c} / E_{0}$ to the ratio $r_{0}$ [see $[12]$ ], of typical value 0.02 , is a purely PV-conserving signal, which does not affect the determination of the PV-related effect. The latter is determined through measurements of the change in $r_{0}$ with polarization angle $\theta$. Second, the signal directed to the lock-in measuring $R_{0}^{[1]}$ is filtered with an amplified band-pass filter, which provides a gain of $101.67(22)$ for the $1^{\text {st }}$ harmonic while attenuating the $2^{\text {nd }}$ harmonic $\approx 50$ times. These two steps result in $R_{0}^{[1]}$ and $R_{0}^{[2]}$ signals of comparable size presented to the respective lock-in amplifiers. Finally, to avoid potential systematic effects due to the changing signal levels when measuring different isotopes, a variable-gain amplifier is used to adjust the signal level at the output of the detection-region photodetector. The gain values in this amplifier are related to the different isotopic abundances of the four $\mathrm{Yb}$ isotopes measured, such that the same signal level is always presented to the lock-ins, regardless of isotope measured.

\section{INVESTIGATION OF SYSTEMATIC EFFECTS AND RELATED ERRORS}

In this section we present a detailed analysis of systematic contributions and uncertainties related to the isotopic comparison measurements. These uncertainties are either due to the limited accuracy of the various calibrations or imperfect estimates of the contribution of PVmimicking effects. We begin by discussing the various $\mathrm{PV}$-data calibrations and the errors in these, since the latter dominate the total systematic uncertainty in the present experiment. We then present an analysis of falsePV contributions and the related uncertainties. Finally, auxiliary experiments done to ensure consistency with our model of harmonics ratios, as well as to investigate potentially unaccounted-for systematics, are discussed at the end of the section.

\section{A. Calibrations to PV-data and related uncertainties}

\section{1. $408 \mathrm{~nm}$ transition saturation}

In the absence of saturation in the Stark-induced transition, the $408 \mathrm{~nm}$ signal grows as $E^{2}$. In the present experiment the transition is weakly saturated. This slight saturation affects the measurement of the harmonics ratio $r_{0}$, and a correction needs to be made. The transition rate can be generally expressed as [50]:

$$
R=\frac{k E^{2}}{1+\frac{E^{2}}{E_{s}^{2}}} .
$$

The parameter $k$ is an overall constant (which depends on the light power in the $\mathrm{PBC}$ ), $E_{s}$ the saturation electric field, and $E=E_{0} \cos \omega t+\zeta / \beta$ includes the applied electric field and the effective electric field $\zeta / \beta$ that results from the PV $\left(|\zeta / \beta|<<E_{0}\right)$. The field $E_{s}$ depends on the intensity of the $408 \mathrm{~nm}$ light exciting atoms. The rate $R$ is saturated when $E_{0}$ becomes comparable to $E_{s}$. In the present experiment, the $408 \mathrm{~nm}$ transition in the atomic beam is weakly saturated $\left(E_{0} / E_{s} \approx 0.1\right)$. To quantify the impact on the harmonics ratio, we expand $R$ in terms of the parameter $\left(E_{0} / E_{s}\right)^{2}$, and compute $r_{0}$. To first order in this parameter, the modified ratio is:

$$
r_{0}=\frac{\zeta}{\beta E_{0}}\left(1-\frac{1}{2} \frac{E_{0}^{2}}{E_{s}^{2}}\right) .
$$

$\mathrm{PV}$ data need to be therefore divided by:

$$
C_{s}=1-\frac{1}{2} \frac{E_{0}^{2}}{E_{s}^{2}} .
$$

Similar analysis shows that the $2^{\text {nd }}$ harmonic in the transition rate is also diminished in the presence of saturation, by a factor $\left(1-E_{0}^{2} / E_{s}^{2}\right)$.

In the presence of transition saturation, harmonics higher than the $2^{\text {nd }}$ emerge in the rate of eq. 15 . We make use of a $4^{\text {th }}$ harmonic amplitude to measure the saturation parameter $E_{s}$. The ratio of $4^{\text {th }}$ to $2^{\text {nd }}$ harmonic amplitudes (to first order in $\left.\left(E_{0} / E_{s}\right)^{2}\right)$ is given by $E_{0}^{2} / 4 E_{s}^{2}$. Measurements of this ratio with varying $E_{0}$ (in the range $1-2.5 \mathrm{kV} / \mathrm{cm}$ ) are made to determine $E_{s}$.

We show in fig. 4 measurements of the parameter $E_{s}$, made in each of the 34 days in which actual isotopic comparison PV-data were acquired. A periodic pattern can be observed in the data that involves a gradual decrease in $E_{s}$, followed by a recovery. This effect is currently not fully understood; however, as we observe, it is generally correlated with gradual deterioration of the in-vacuum 


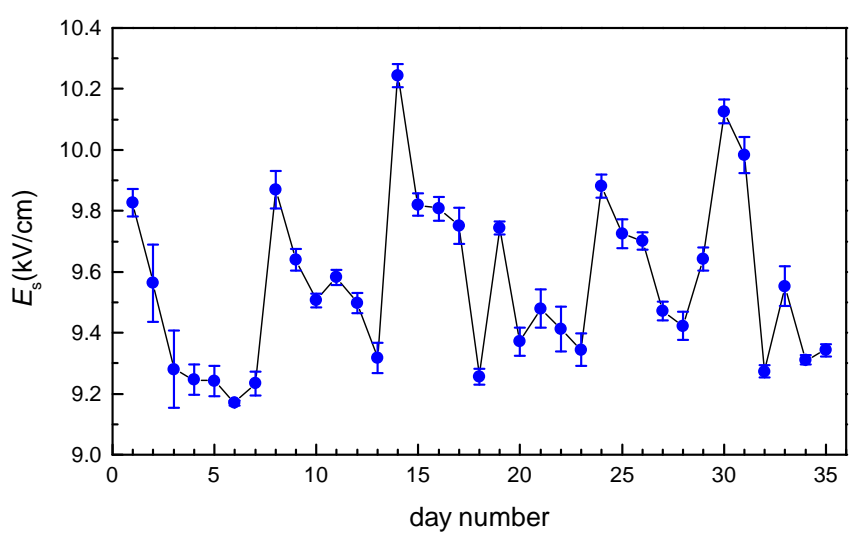

FIG. 4. (Color online) Measurements of the saturation electric field for the $0 \rightarrow 0$ component of the $408 \mathrm{~nm}$ transition, made on each of the 34 days in which PV-data where acquired. Each data point represents the average of four measurments, two of which were made for angle $\theta_{+}=+\pi / 4$ and another two for $\theta_{-}=-\pi / 4$.

PBC mirrors, in the presence of the intense near-UV light. Typically, operation of the PBC for a few days results in a decrease in the cavity finesse and power buildup of about $30 \%$. The gradual decrease in $E_{s}$ should be occurring due to an increase in the intra-cavity circulating power (which corresponds to an increase in the degree of saturation in the transition rate). Since the power transmitted through the PBC is actively stabilized, the observed effect implies that the transmission of the cavity output coupler gradually decreases. Recovery of the cavity mirrors is possible by exposing them to partial atmosphere (tens of mbar) for $\approx 1 \mathrm{~min}$, in the presence of the intense $408 \mathrm{~nm}$ light. The recovery process generally results in an increase of $E_{s}$. As seen in fig. 4), the saturation field $E_{s}$ increases following venting of vacuum system which was done to recover PBC mirror performance before days \# 1, 8, 14, 19, 24, 30. We assume an error of $3 \%$ in the daily $E_{s}$ value, to take into account possible drifts of this parameter over the 8-16 hr long PV-run.

\section{Polarization parameter $p$}

The $408 \mathrm{~nm}$ polarization parameter $p$ of eq. (14) needs to be precisely measured for an accurate $\zeta / \beta$ determination. For angles in the range $\left|\theta_{ \pm}\right|=\pi / 4 \pm 0.02$ and $\left|\phi_{ \pm}\right| \leq 0.06$, this parameter can be approximated (with an error of a few parts per $10^{5}$ ) as $p \approx p_{\theta} \cdot p_{\phi}$, with:

$$
\begin{aligned}
& p_{\theta}=\cot \theta_{+}-\cot \theta_{-}, \\
& p_{\phi}=\cos \phi_{+}-\cos \phi_{-} .
\end{aligned}
$$

This separation of variables simplifies the determination of $p$. In the following, we discuss how $p_{\theta}$ and $p_{\phi}$ are measured.
Continuous measurements of $\theta$ during PV data acquisition are made with the PBC polarimeter described in section III. Prior to commencing an acquisition run, a calibration of the PBC polarimeter is required. To perform this calibration, measurements of the relative sizes of the three transition components in the $408 \mathrm{~nm}$ spectrum (see fig. $3 \mathrm{~b}$ ) are used to read the intracavity light polarization angles $\theta_{ \pm}$(nominally $\pm \pi / 4$ ); these angles are correlated with the concurrent readings the PBC polarimeter, thereby providing a calibration of the polarimeter. Subsequent measurements of the light transmitted through the $\mathrm{PBC}$ during a many-hour-long PV run provide an accurate tracking of the angles $\theta_{ \pm}$. A detailed description of the method to determine the initial $\theta_{ \pm}$angles using the atoms as polarization probes, including the effects of apparatus imperfections, is given in Appendix $B$.

The uncertainty in $p_{\theta}$ has two contributions: the statistical uncertainty associated with the initial $\theta_{ \pm}$measurement using the atoms, and the systematic uncertainty arising from drifts in the readings of the polarimeter at the output of the PBC over a many-hour period. The statistical uncertainty (typically $<0.1 \%$ of the PV effect) is added in quadrature with the statistical error in a block of data acquired in a daily run. To make an estimate for the systematic uncertainty, we took two long sets of polarization data. In these runs, following the initial correlation of the $\theta_{ \pm}$readings with the polarimeter readings, the $p_{\theta}$ measurements made with the two methods were compared over a period of 12 hours. These data are presented in fig. 5. During these runs, the $\mathrm{PBC}$ was unlocked several times, to investigate the effect of thermal cycles of the PBC optics on the actual polarization angle (read with the atoms), and well as on its measurement with the polarimeter. The data show that unlocking the $\mathrm{PBC}$ for minute-long periods of time, does have an impact on the intra-cavity polarization angle (fig. 5a). These polarization shifts are nevertheless tracked well by the polarimeter, as seen in fig. 5 b. The relative drifts between the $p_{\theta}$ determinations made using the $408 \mathrm{~nm}$ resonance profile and those made using the polarimeter are always less than $10^{-3}$ of the nominal value $p_{\theta}=2$. We assign a $10^{-3}$ fractional systematic uncertainty in determining $p_{\theta}$.

The light ellipticity-related parameter $p_{\phi}$ is determined through measurements made using signals from the atoms. The idea is to observe a term in the harmonics ratio of the $m^{\prime}= \pm 1$ components of the $408 \mathrm{~nm}$ transition, that has a dependence on the angle $\phi$. Expressions for the excitation rate for these components, as well as the corresponding harmonics ratios $r_{+1}$ and $r_{-1}$ in the presence of field imperfections, are given in Appendix A. The difference $r_{+1}-r_{-1}$ (retaining terms up to $2^{\text {nd }}$ order in the various field imperfections), is given by:

$$
r_{+1}-r_{1}=\frac{8 e_{z}}{E_{0}} \tan \theta \sin \phi
$$

A measurement of $r_{+1}$ and $r_{-1}$ with an enhanced field 


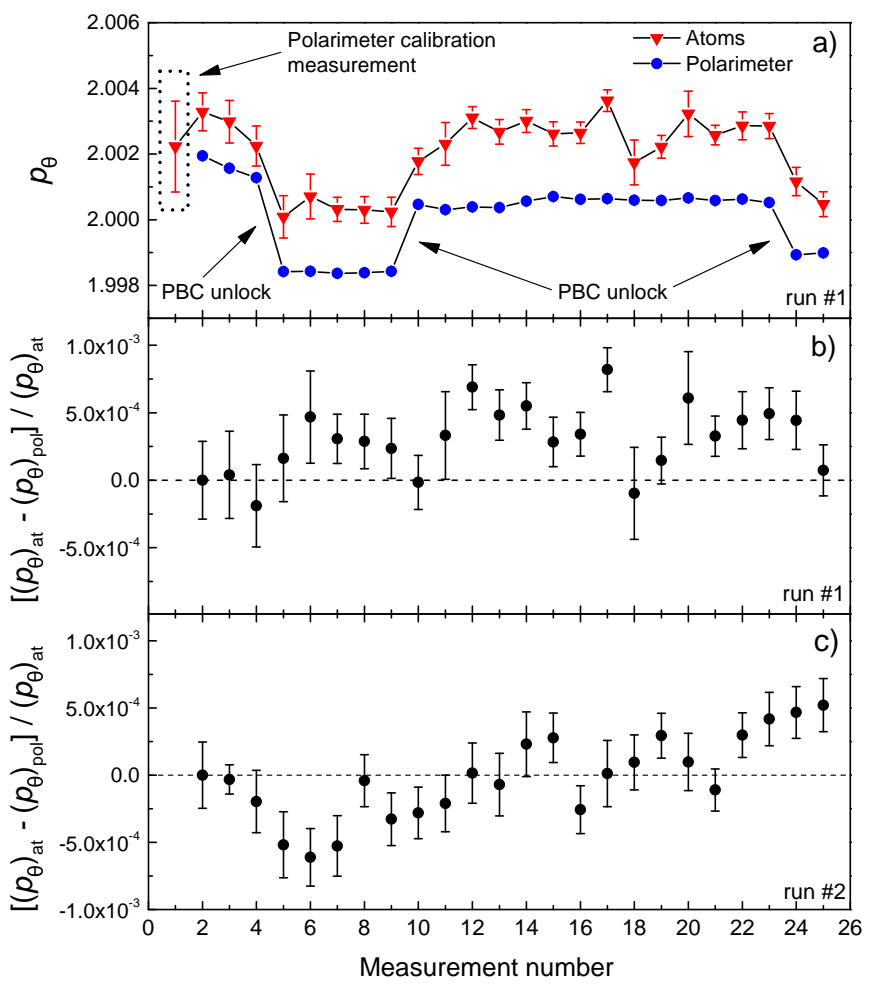

FIG. 5. (Color online) a) Comparison of $p_{\theta}$ measurements made with atoms and those made with the PBC polarimeter, over a $12 \mathrm{hr}$ period. Error bars (smaller than data points for the polarimeter data) represent standard errors of the corresponding mean values. The first data point in the plot represents the initial reading of angles $\theta_{ \pm}$using the $408 \mathrm{~nm}$ spectrum. These readings are used to assign an initial value to the corresponding polarimeter readings. The statistical error in this first $p_{\theta}$ measurement explains the relative offset between the 'atoms' and 'polarimeter' points in the second measurement (points with measurement \#2). The polarimeter calibration measurement (measurement \#1) has greater error than subsequent measurements, as it has smaller integration time compared to the time devoted to measure subsequent points. The sub- $0.1 \%$ statistical error of this calibration measurement is negligible compared to the $\sim 1 \%$ statistical error of a daily block of PV data. The PBC was unlocked several times, with the duration of each pause in the range 5-10 min. Shorter ( $\approx 10 \mathrm{~s})$ interruptions in the PBC lock were also made, and have no visible impact on polarization. b) Relative difference in $p_{\theta}$ readings between the two methods for the data shown in a). The offset between the 'atoms' and 'polarimeter' values at the start of the run ( see points with measurement \#2 in plot a)), is of statistical nature, and is removed in b), to allow for a study of relative drifts between the two determinations. c) Results of $p_{\theta}$ differences measured in another $12 \mathrm{hr}$-long run.

component $e_{z},\left(e_{z} / E_{0} \approx \pm 0.06\right)$ allows for extraction of $\phi_{+}$or $\phi_{-}$, corresponding to polarization states with $\theta_{+}$ or $\theta_{-}$respectively. The overall accuracy is determined by statistics (the ratio $e_{z} / E_{0}$ is known to within $1 \%$, and $\tan \theta$ is measured with sub- $1 \%$ uncertainty).

Measurements of $\phi_{ \pm}$were made before the start and

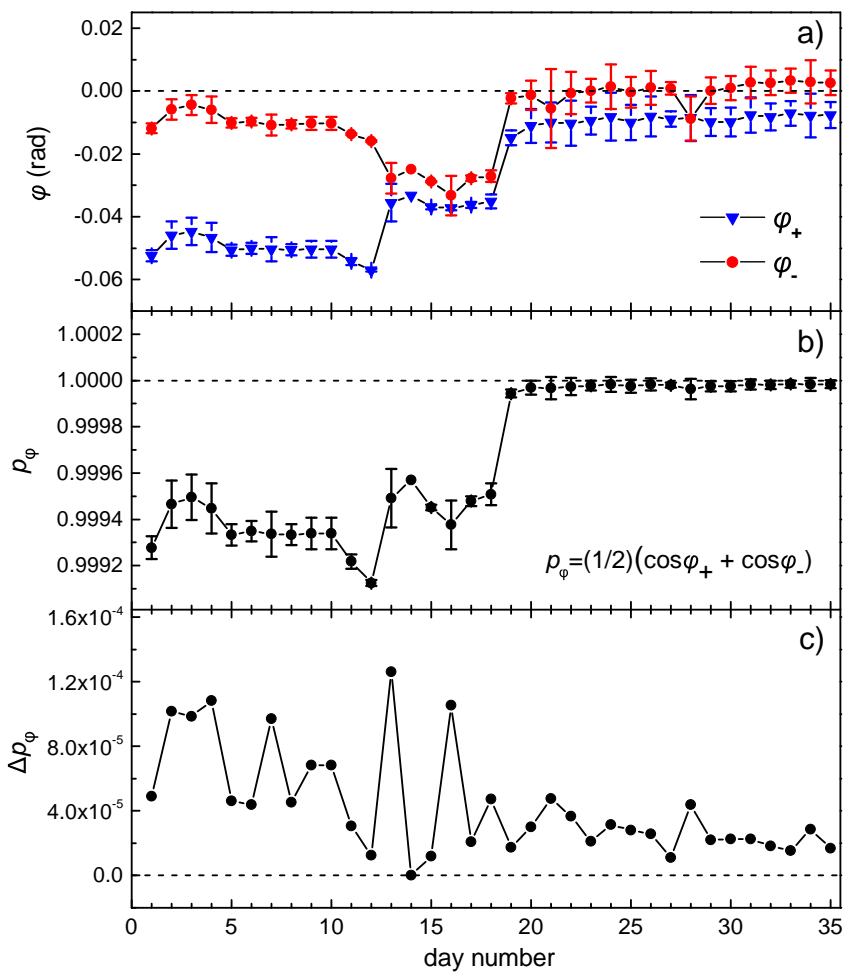

FIG. 6. a) (Color online) Angles $\phi_{+}$and $\phi_{-}$determined on each of the 34 days of actual PV-data acquisition. Each data point represents the mean value of two measurements, of which the first was done before the start of the PV-run, and the second after the end of the run. The error bars correspond to the standard deviation of the mean. The changes in these values on days \#13 and \#18 are due to a readjustment of the tilt of the half-wave plate that controls the 408 $\mathrm{nm}$ light polarization coupled to the PBC. b) Corresponding $p_{\phi}$ parameter for the values shown in a). Error bars come from propagation of errors in the data points of a). c) Plot of the error-bar values for the data shown in b). The maximum error is $1.2 \cdot 10^{-4}$.

after the end of each of the many-hour-long PV-runs, employing both orientations of the magnetic field $B_{z}$. The $\phi_{ \pm}$value for the corresponding daily block of PV-data is taken as the mean of the initial and final measurements, and the error assigned to this mean is the standard deviation of the two values. We show the results of these measurements as well as resulting parameter $p_{\phi}$ and the error in its determination in fig. 6. We assign a fractional error of $1.2 \cdot 10^{-4}$ in the determination of $p_{\phi}$. This is a negligible contribution to the overall error in the polarization parameter $p$, an error dominated by the $10^{-3}$ fractional error in $p_{\theta}$.

\section{Effect of partial peak overlap}

The applied magnetic field in the interaction region results in a resolved spectrum for the ${ }^{1} \mathrm{~S}_{0} \rightarrow{ }^{3} \mathrm{D}_{1}$ transition (fig 3). A small residual overlap between the different 
peaks is still present, however, and its effect on the PVmeasurements needs to be considered. In the presence of the overlap, the transition rate at the spectral peak position of the $0 \rightarrow 0$ transition component (where the $\mathrm{PV}$-data are acquired) is given by:

$$
R_{0}^{\prime}=R_{0}+h \cdot R_{-1}+h \cdot R_{+1} .
$$

The terms $R_{-1}$ and $R_{+1}$ are the rates of the $0 \rightarrow-1$ and $0 \rightarrow+1$ components, and $h$ is a parameter that quantifies the contribution of the wing of a peak to the signal of the adjacent peak, measured to be $h=4.2(4) \cdot 10^{-4}$. In formulating the total rate $R_{0}^{\prime}$ in eq. 21), quantum interference between the transition amplitudes of the different Zeeman sub-levels is not considered. Such an effect does not take place in our system, since the emitted fluorescence light from de-excitation of atoms has different polarizations for the three excited-state sublevels. Because of this, the corresponding excitation paths $\left(m=0 \rightarrow m^{\prime}=0, \pm 1\right)$ can be distinguished and amplitude interference does not occur. The resulting harmonics ratio $r_{0}^{\prime}$ can be computed from 21, and from that, the corresponding combination $K_{1}^{\prime}$ (Table I), can be determined:

$$
K_{1}^{\prime}=\frac{16 \zeta}{\beta}(1-2 h)
$$

To derive 222, field imperfections, which generally have a greater impact on the PV-measurements in the $0 \rightarrow \pm 1$ transitions, were neglected. This is a reasonable simplification. As discussed in section IV C2, the PVeffect on the $0 \rightarrow \pm 1$ transitions, is, to within $2 \%$, consistent in magnitude with the effect measured on the $0 \rightarrow 0$ transition. An additional $2 \%$ correction to the small calibration parameter of order $h$, would have a negligible impact on the PV-measurements taken on the $0 \rightarrow 0$ component. The negative sign in the signal contribution from the $0 \rightarrow \pm 1$ transitions is expected, since the PVeffect for these transitions is of opposite sign compared to that of the $0 \rightarrow 0$ component (see Appendix A). To correct for the effect of the patial overlap of the different Zeeman components in the ${ }^{1} \mathrm{~S}_{0} \rightarrow{ }^{3} \mathrm{D}_{1}$ transition, the PVmeasurements are divided by a factor $C_{\text {overlap }}=(1-2 h)$.

\section{Transit time from interaction to detection region}

Due to the time required for excited atoms to reach the detection region and be measured, there is a phase delay in the detected excitation rate, relative to the electric field phase in the interaction region. This would not be an issue for a beam of atoms all moving with the same longitudinal velocity; however, because of the longitudinal velocity spread in the atomic beam, atoms in different velocity classes are detected at different times. This leads to a slight mixing of phases in the measured rate for these different classes, and to a frequency-dependent attenuation of the amplitude of each harmonic. The result of this attenuation is a detected harmonics ratio $r_{0}$ that is slightly larger than the actual one. This effect was modeled in 38. We correct for it by dividing the measured $r_{0}$ by a factor $C_{\text {transit }}=1.00285(10)$. This factor is an order of magnitude lower than that in [38. The reduction is due to the lower electric-field frequency $(19.9 \mathrm{~Hz})$ in the present experiment, compared to that of the previous one $(76 \mathrm{~Hz})$. The assigned error in $C_{\text {transit }}$ comes from the assumed uncertainty in the temperature of the $\mathrm{Yb}$ oven $\left( \pm 50^{\circ} \mathrm{C}\right)$ and from the assumed $0.5 \mathrm{~cm}$ uncertainty in the distance between the interaction and detection regions. The expected phase-delays in $1^{\text {st }}$ and $2^{\text {nd }}$ harmonic signals present in the transition rate $\left(-4.8^{\circ}\right.$ and $\left.-9.6^{\circ}\right)$ are detected correctly, to within $0.5^{\circ}$. A $0.5^{\circ}$ error in the detected phase of a given harmonic in the excitation rate, would result in a fractional decrease of $5 \cdot 10^{-5}$ in the measured harmonic amplitude. The uncertainties in the measured PV effect arising from such small phase uncertainties in detecting the $1^{\text {st }}$ and $2^{\text {nd }}$ harmonics, are negligible.

\section{Photodetector response calibration}

The detection-region photodetector (PD) has a finite bandwidth, measured to be $1.1 \mathrm{kHz}$. The PD low-passfilter behavior at the $1^{s t}$ - and $2^{\text {nd }}$ - harmonic frequencies present in the transition rate $(19.9 \mathrm{~Hz}$ and $39.8 \mathrm{~Hz}$, respectively) is expected to have an impact on the measured ratio $r_{0}$. To quantify this impact, we measured the frequency-dependent response of the $\mathrm{PD}$, relative to that of a fast photodetector (Thorlabs PDA100, $220 \mathrm{kHz}$ bandwidth). Using a light-emitting-diode as a source of sinusoidally modulated light, we measured with the PD a ratio of amplitudes at $39.8 \mathrm{~Hz}$ and $19.9 \mathrm{~Hz}$, which was $1.00040(17)$ times greater than the ratio determined with the fast detector. The error in the measured amplitudes ratio is mainly statistical. The measured $r_{0}$ values are scaled down by $C_{P D}=1.00040(17)$ to compensate for the $\mathrm{PD}$ finite response time.

\section{6. $P D$ signal conditioning calibration}

There is an overall calibration factor $C_{e}$ relating the harmonics-ratio value recorded in the laboratory $\mathrm{PC}$ to the actual ratio at the output of the PD. This factor needs to be precisely measured. As part of the effort to improve detection conditions for the small $1^{\text {st }}$-harmonic signal in the transition rate, the PD signal is bandpassamplified and then measured with a lock-in amplifier (see section III). The $1^{\text {st }}$-harmonic reading is recorded in the computer, as is the reading from another lock-in that measures the $2^{\text {nd }}$ harmonic directly at the PD output. The calibration factor $C_{e}$ was measured by replacing the $\mathrm{PD}$ with an electronic circuit that adds two known sig- 
nals at the $\omega$ and $2 \omega$ frequencies. This circuit attenuates the $\omega$ signal to simulate the amplitude level in the actual experiment. The transfer function of this circuit for each of the two signal paths was measured at the $10^{-4}$ level. The inputs to the circuit come from a dual-channel function generator (Keysight 33510B) and are measured with a laboratory multimeter (Keysight 34410A), whose measurements agree with those made with an identical unit, at the $10^{-4}$ level. A comparison of the known harmonics ratio at the output of the adder-circuit, to the reading in the computer, determines $C_{e}$.

Many different measurements of $C_{e}$ where made, with varying signal sizes as well as phase-delays between the lock-in reference phases and the corresponding detected phases. These measurements were carried out twice: before the start of the PV-data acquisition campaign, and after its end. The first measurement yielded a value $C_{e}=101.52(5)$ and a second a value $C_{e}=101.82(1)$. We assign the value of $101.67(22)$ to $C_{e}$, which is the mean of the two results. The $0.22 \%$ error in $C_{e}$ is the standard deviation of the two measurements.

This inadvertent drift in the $C_{e}$ calibration gives rise to the main systematic uncertainty in this experiment. Since the PV-data were acquired in a pattern that involved alternating measurements between isotopes, however, the impact of this drift on the actual isotopic comparison should be minimal.

\section{Electric-field calibration}

Accurate knowledge of the electric field applied to the atoms is needed to relate a determination of $K_{1}$ (see Table Il) to the ratio $\zeta / \beta$. There are two dominant uncertainties in the electric field. The first is an uncertainty in the calibration of the voltage monitor outputs in the two high-voltage amplifiers (model TREK 609B), used to apply voltage to the main field plates. The corresponding error in the applied voltage is a fractional $6 \cdot 10^{-4}$. The second uncertainty comes from imperfections in the construction of the field-plate system and the finite accuracy in measuring the field-plate spacing. This spacing was measured at several different places with a precision micrometer. The variation in the mean spacing (5.5045 $\mathrm{cm})$ was found to be $0.002 \mathrm{~cm}$, which corresponds to a fractional uncertainty in the spacing of $\approx 4 \cdot 10^{-4}$, and to the same contribution to the overall electric field error.

\section{B. False-PV signals and related uncertainties}

In this subsection we discuss the methods to study and control known systematic contributions to the measurements which mimic the PV effect.

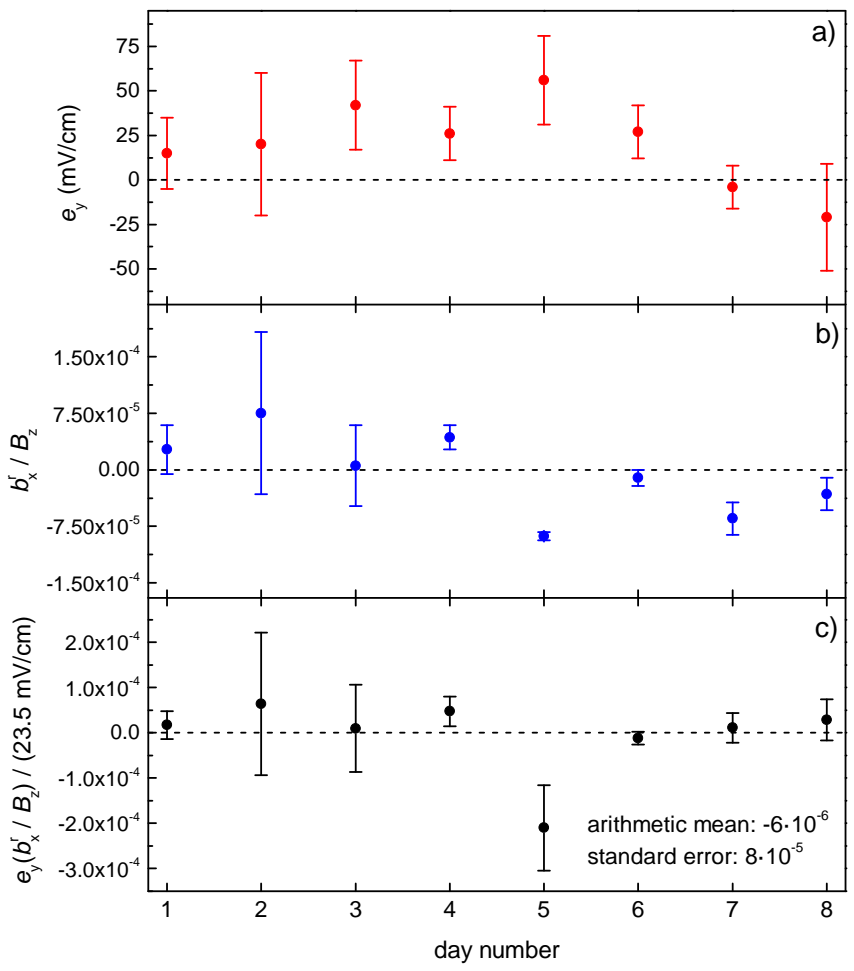

FIG. 7. (Color online) a) Measurements of the stray field $e_{y}$, made regularly during the isotopic comparison PV-run. This field is always $75 \mathrm{mV} / \mathrm{cm}$ or less in magnitude. b) Measured residual $b_{x}^{r} / B_{z}$ values. The compensated ratio is stable at the $10^{-4}$ level. c) Fractional contribution of the $e_{y} b_{x}^{r} / B_{z}$ term to the measurement of the PV effect.

\section{1. $e_{y} b_{x}^{r}$ contribution}

Examination of the combination $K_{1}$ (Table I), shows that the coupling of a stray $e_{y}$ field to the reversing magnetic field component $b_{x}^{r}$ gives rise to the false-PV contribution proportional to $e_{y} b_{x}^{r} / B_{z}$, which directly competes with $\zeta / \beta$. The strategy to handle this contribution is to minimize $b_{x}^{r}$, and then measure the residual effect periodically during the PV data acquisition, and, if needed, apply a correction to the PV-data.

To measure $b_{x}^{r}$ we apply an enhanced $e_{y}$ field, with $e_{y} / E_{0}= \pm 0.062(1)$, and observe the change in $K_{1}$ as the polarity of this enhanced field is reversed. This allows one to isolate the $e_{y} b_{x}^{r} / B_{z}$ term and measure $b_{x}^{r} / B_{z}$. The typical value for this misalignment is $1-2 \cdot 10^{-3}$. We then use shimming coils to to apply a reversing field to null $b_{x}^{r}$. With this procedure the residual $b_{x}^{r} / B_{z}$ ratio is measured to be $10^{-4}$ or less. We find that this cancellation is very stable with time (over month-long periods). Readjustment is only required when the alignment of the $\mathrm{PBC}$ optical axis (that defines the $\mathrm{x}$-axis in the coordinate system) is changed. Such a change was only made once during the isotopic comparison data run.

With a suppressed $b_{x}^{r} / B_{z}$ ratio, one has to monitor $e_{y}$ during the PV-data campaign. To measure $e_{y}$ we make 
use of the combination $K_{3}=-16 e_{y} b_{x} / E_{0} B_{z}$. Another set of coils is used to apply an enhanced $b_{x}$, with $b_{x} / B_{z} \approx$ $\pm 0.0390(6)$. Observation of the variation of $K_{3}$ with a sign flip in $b_{x} / B_{z}$ is used to determine $e_{y}$.

We show measurements of $e_{y}$ and the residual $b_{x}^{r} / B_{z}$ ratio in fig. 7. These measurements were made concurrently, at regular intervals during the isotopic comparison $\mathrm{PV}$ run. The term $e_{y} b_{x}^{r} / B_{z}$ was never greater than $2 \cdot 10^{-4}$ of the measured PV-effect. The (arithmetic) mean value of the systematic is smaller than $10^{-5}$. The error (standard error of the arithmetic mean) is less than $10^{-4}$ of the PV effect. We conclude that the contribution of this systematic to the PV measurements is negligible. We did not make use of weights in this statistical analysis, since the results of fig. 7 come from short acquisition runs, therefore the corresponding error bars may not represent errors accurately.

\section{2. $d K_{1} / d b_{x}$ systematic}

The $e_{y} b_{x}^{r} / B_{z}$ term is the only parasitic contribution in $K_{1}$, within our model for the harmonics ratio, and up to $2^{\text {nd }}$ order in field imperfections. During auxiliary experiments that involved consecutive application of all possible field imperfections to the atoms, as a check for unaccounted-for systematic contributions, we discovered a dependence of $K_{1}$ on the non-reversing $b_{x}$ component of the magnetic field. $K_{1}$ changes with $b_{x}$ at a rate of $\approx$ $3 \% / \mathrm{G}$, for the $B_{z}=93 \mathrm{G}$ leading field. The origin of this effect is currently not understood. We did investigate its dependence on other parameters. No dependence was found on applied non-reversing or reversing electric-field components, or the amplitude of the leading electric field $E_{0}$. We did observe a $\cot \theta$ dependence of the effect, like the PV effect itself has.

This spurious effect is periodically measured and corrections to the $\mathrm{PV}$-data are made. To measure the residual $b_{x}$ field, we make use of $K_{3} \approx-16 e_{y} b_{x} / B_{z} E_{0}$, in a manner similar to that described earlier for the measurements of the $e_{y}$ field. Here we apply a known $e_{y}$, so that $e_{y} / E_{0} \approx \pm 0.0644(10)$, and observe the change in $K_{3}$, correlated with a polarity flip in $e_{y}$. In measurements made periodically during the $\mathrm{PV}$-data run campaign, the observed $b_{x}$ values were always smaller than $20 \mathrm{mG}$.

We show the measured dependence of $K_{1}$ on $b_{x}$ in fig. 8 a, along with calibration measurements of the spurious effect in fig $8 \mathrm{~b}$. These data were taken regularly during the PV-data acquisition. The corresponding fractional $K_{1}$ change, inferred from the data of a) and b), is shown in fig. 8. The arithmetic mean value of the change is $2.6 \cdot 10^{-4}$. We subtract this fraction from all PV data to account for this systematic effect, and assign a fractional uncertainty $2 \cdot 10^{-4}$, which represents the standard error of the arithmetic mean value. As in the studies related to the $e_{y} b_{x}^{r} / B_{z}$ systematic, use of weights in the statistical analysis is avoided.

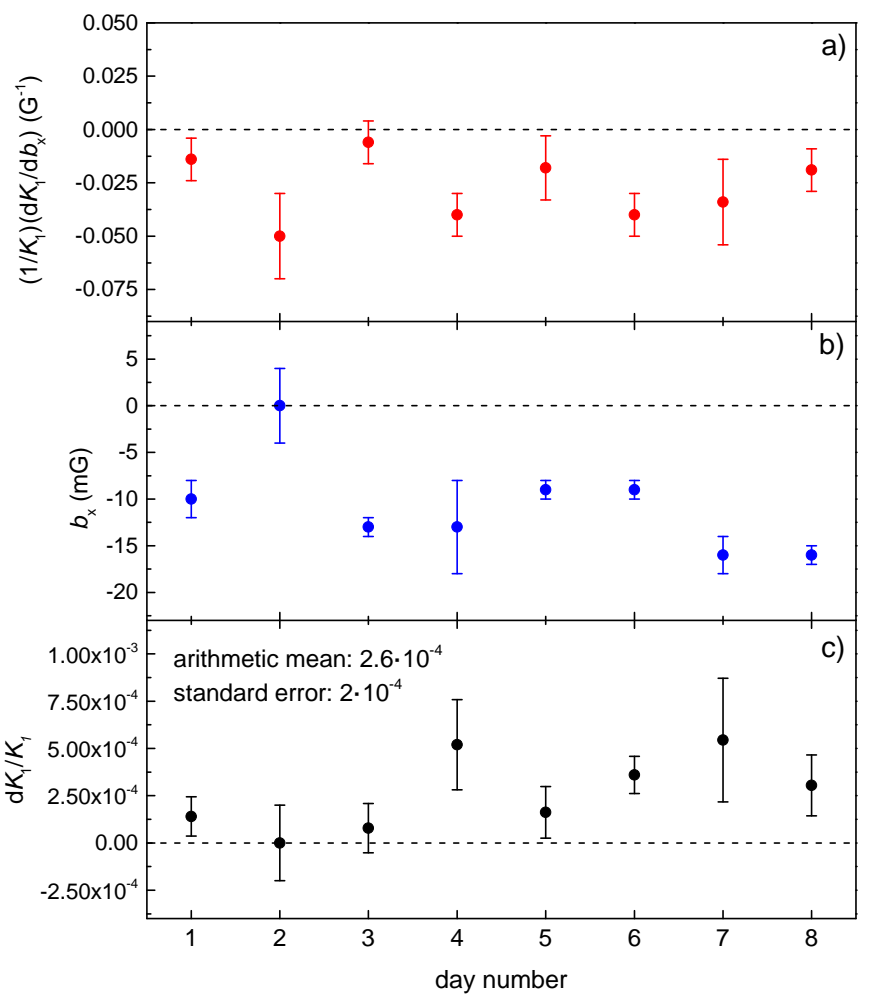

FIG. 8. (Color online) a) Fractional change in $K 1$ per $\mathrm{G}$ of the applied field $b_{x}$. b) Measured residual $b_{x}$ field. c) Fractional change in $K 1$, inferred from the data of a) and b).

\section{3. $E_{d c}$ and transition saturation}

The $E_{\mathrm{dc}}$ field, applied to improve conditions in the Stark-PV interference detection, gives rise to a false-PV signal in the presence of saturation in the transition. To illustrate this, we consider the harmonics ratio of eq. (8):

$$
r_{0}\left(\theta, f_{B}\right)=C_{s}\left(\frac{4 E_{d c}}{E_{0}}+\frac{4 \zeta}{\beta E_{0}} \cot \theta\right)
$$

where $C_{s}$ represents the slight saturation-related reduction in $r_{0}$ and is given by 17 ). This factor depends on the overall excitation rate. The corresponding combination $K_{1}$ is given by:

$K_{1}=\frac{16 E_{d c}}{E_{0}}\left(C_{s+}-C_{s-}\right)+\frac{8 \zeta}{\beta E_{0}}\left(\cot \theta_{+} C_{s+}-\cot \theta_{-} C_{s-}\right)$.

The saturation factors $C_{s+}$ and $C_{s-}$ correspond to the two angles $\theta_{+}$and $\theta_{-}$, respectively, and are generally slightly different, due to unequal excitation rates for the angles $\theta_{+}$and $\theta_{-}$. Unequal excitation rates occur because $\theta$ is not precisely set to either $+\pi / 4$ or $-\pi / 4$. The quantity in parenthesis in the second term of (19) can be approximated as:

$$
\frac{1}{2}\left(C_{s+}+C_{s-}\right)\left(\cot \theta_{+}-\cot \theta_{-}\right)
$$


with an accuracy at the $10^{-5}$ level for angles $\theta_{ \pm} \approx \pm \pi / 4$ and the typical value $\approx 0.01$ for $C_{s+}$ and $C_{s-}$. The parameter $K_{1}$, from which the $\mathrm{PV}$-related parameter $\zeta / \beta$ is extracted, can be then expressed as:

$$
\begin{aligned}
K_{1}= & \frac{16 E_{d c}}{E_{0}}\left(C_{s+}-C_{s-}\right) \\
& \quad+\frac{1}{2}\left(C_{s+}+C_{s-}\right) \frac{8 \zeta}{\beta E_{0}}\left(\cot \theta_{+}-\cot \theta_{-}\right) .
\end{aligned}
$$

Since $\left|C_{s+}-C_{s-}\right| \neq 0$, extraction of $\zeta / \beta$ from $K_{1}$ is influenced by the presence of the first term in 25$)$. This term is linear in $E_{d c}$ and leads to a fractional false-PV systematic:

$$
\frac{E_{d c}}{\zeta / \beta}\left|C_{s+}-C_{s-}\right|
$$

During a PV-run we observe excitation rates for the two polarization angles $\theta_{ \pm}$which typically differ by $\approx$ $0.5 \%$. This is because these angles are not precisely $\pm \pi / 4$. Given that the saturation electric field $E_{s}$ (17) grows as the square root of the signal, we can estimate that for the typical $E_{0}=1 \mathrm{kV} / \mathrm{cm}$ and $E_{s}=10 \mathrm{kV} / \mathrm{cm}$, the quantity $\left|C_{s+}-C_{s-}\right| \approx 2.5 \cdot 10^{-5}$. Using the value of $\left|E_{d c}\right|=6.3 \mathrm{~V} / \mathrm{cm}$ of this experiment, and the measured $|\zeta / \beta| \approx 23.5 \mathrm{mV} / \mathrm{cm}$, we find that the false-PV term of 26 is a fractional $0.7 \%$.

We handle this systematic by averaging PV-data taken with opposite $E_{d c}$ polarities. The more precisely $E_{d c}$ is reversed, the better the suppression of the related systematic. A good reversal is achieved with feedback on the $E_{d c}$ value. To implement this, we make use of the combination $K_{4}$ (see table I), which, to an excellent approximation, is equal to $16 E_{d c} / E_{0}$ (other terms in $K_{4}$ are suppressed by at least $10^{4}$ times relative to this term). While data are being acquired, $K_{4}$ is monitored. Every time the $E_{d c}$ polarity is flipped to negative, an adjustment is made to the new $E_{d c}$ setting, to correct for small differences between the magnitudes of the previous two $K_{4}$ measurements, one of which corresponds to $E_{d c}>0$ and the other to $E_{d c}<0$. As a result, the total static field along $x$ (i.e. the sum of the $\left|E_{d c}\right| \approx 6.3 \mathrm{~V} / \mathrm{cm}$ field and a stray field) is reversed to within $5-10 \mathrm{mV} / \mathrm{cm}$, leading to a practically complete suppression of the related systematic effect.

We note that the slight mismatch between the saturation-related parameters $C_{s+}$ and $C_{s-}$ does not affect the determination of the calibration factor $(1 / 2)\left(C_{s+}+C_{s-}\right)$ in 25$)$. This factor is determined as the average of measurements of the parameter $C_{s}$ made on both angles $\theta_{+}$and $\theta_{-}$.

\section{C. $\zeta / \beta$ sign and consistency checks}

In this sub-section we discuss observations made to establish the sign of $\zeta / \beta$. The present determination disagrees with that of the 2009 experiment [37, 38, which we have traced to a sign error in the analysis code employed in that work. We also provide the results of auxiliary experiments done to ensure consistency between measurements and our model for the expected PV effect.

\section{1. $\zeta / \beta$ sign determination}

The primary method to determine the sign of $\zeta / \beta$ is to study the $\operatorname{sign}$ of the term $(\zeta / \beta) \cot \theta$ in the harmonics ratio of 12 , in relation to the signs of other terms in this ratio. The latter signs are unambiguously defined once the directions of the fields in the relevant terms are known. The present discussion follows that of 11 . We compare the sign of the PV-induced term in $\sqrt{12}$ with the sign of the term that depends on the $E_{d c}$ field as well as the sign of the PV-mimicking term $b_{x} e_{y} \cot \theta$. We consider the harmonics ratio $r_{0}$ of 12 :

$$
r_{0}=\frac{4 E_{d c}}{E_{0}}+\frac{4 \zeta}{\beta E_{0}} \cot \theta+\frac{4 b_{x} e_{y}}{B_{z} E_{0}} \cot \theta,
$$

where $b_{x}$ and $B_{z}$ are the total fields along $\mathrm{x}$ and $\mathrm{z}$, and we have assumed no polarization ellipticity $(\phi=0)$.

The first step in determining the sign of $\zeta / \beta$ is to examine the sign-relationship between the terms $E_{d c} / E_{0}$ and $(\zeta / \beta) \cot \theta$ in the harmonics ratio $r_{0}$. Application of a large and positive $E_{0}$ allows us to adjust the phases of the lock-in amplifiers measuring the $1^{\text {st }}$ and $2^{\text {nd }}$ harmonics in the excitation rate, to obtain positive outputs with maximal magnitude. (The $E_{d c}$ polarity is checked through measurements made directly on the electric field plates.) We retain these phase values in subsequent PV experiments. We further observe that a reversal of $E_{d c}$ results in a reversal of the $1^{\text {st }}$ harmonic sign. With this procedure we establish the convention that $r_{0}>0$ when $E_{d c}>0$ and $r_{0}<0$ when $E_{d c}<0$. We then experimentally check the sign of the extracted term $(\zeta / \beta) \cot \theta$ in relation to the polarization angle $\theta$. We find that for $\theta>0,(\zeta / \beta) \cot \theta<0$ and $(\zeta / \beta) \cot \theta>0$ when $\theta<0$. We show data that illustrate the above observations in fig. 9 .

The above tests are sufficient to determine the sign of $\zeta / \beta$ provided that the polarization angle is set correctly (see coordinate system in fig. 2). To check this, we extract the contribution of the term $\left(b_{x} / B_{z}\right) e_{y} \cot \theta$ in (27). With application of enhanced fields $b_{x}>0$ and $e_{y}>0$ it is seen that $r_{0}>0$ for $\theta>0$ and vice versa $\left(B_{z}>0\right.$ here). As the polarities of the three relevant fields $\left(b_{x}, B_{z}\right.$ and $e_{y}$ ) are confirmed before these measurements, we verify that the angle $\theta$ is set correctly, and therefore $\zeta / \beta<0$. Figure 10 presents data that support these observations.

Additional checks were performed to ensure consistency of our sign determination for $\zeta / \beta$. These included a cross-check measurement of the ratio of the $M 1$ transition moment and $\beta$, which was determined previously in [51, and independent data analysis of the current PVdata by two of us. These checks are described in [1]. 


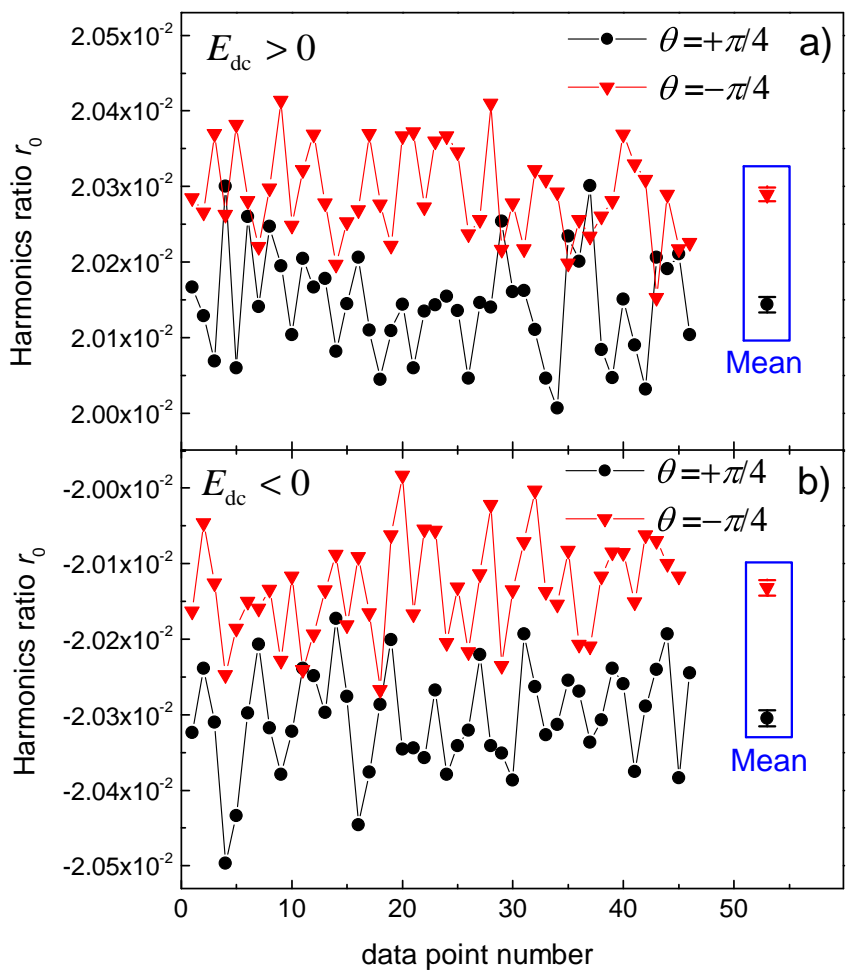

FIG. 9. (Color online) Harmonics ratio $r_{0}$ recorded in ${ }^{172} \mathrm{Yb}$ over a period of $\approx 25 \mathrm{~min}$. Equal number of data points are shown for either orientation of the leading magnetic field $B_{z}$, and polarization angles $\theta_{ \pm} \approx \pm \pi / 4$. In a) the applied $E_{d c}$ field, of approximate magnitude $\left|E_{d c}\right|=6.3 \mathrm{~V} / \mathrm{cm}$, is positive, while in b) it is negative. The ac field applied to the atoms is of amplitude $E_{0} \approx 1218 \mathrm{~V} / \mathrm{cm}$. Observation of the change in $r_{0}$ with the $E_{d c}$-reversal establishes a sign definition for $(\zeta / \beta) \cot \theta$. Assuming $\theta$ is set in a way consistent with its $\operatorname{sign}$ definition (see coordinate system in fig. 2), then the observed dependence of $r_{0}$ on the $\theta$-reversal, is sufficient to determine the $\zeta / \beta$ sign. The angle polarity is checked with measurements presented in fig. 10 The difference in the mean $r_{0}$ ratios of a) and b) is $-2(8) \cdot 10^{-6}$, corresponding to a difference in the applied $E_{d c}$ magnitudes of $-0.6(5) \mathrm{mV} / \mathrm{cm}$, which is indicative of the quality of the $E_{d c}$ reversal in the experiment.

The sign discrepancy between the present results and the previous $\mathrm{Yb}$ PV measurements [37, 38] was traced to a sign-error in the data analysis performed in that work. The procedure to discover the origin of the discrepancy is discussed in the methods section of [1].

\section{Other consistency checks}

In addition to the measurements related to the sign of the PV effect, a number of auxiliary experiments were performed as part of a process to check for unaccountedfor systematics and establish consistency between our model for the harmonics ratio and actual observations under varying apparatus conditions. These experiments

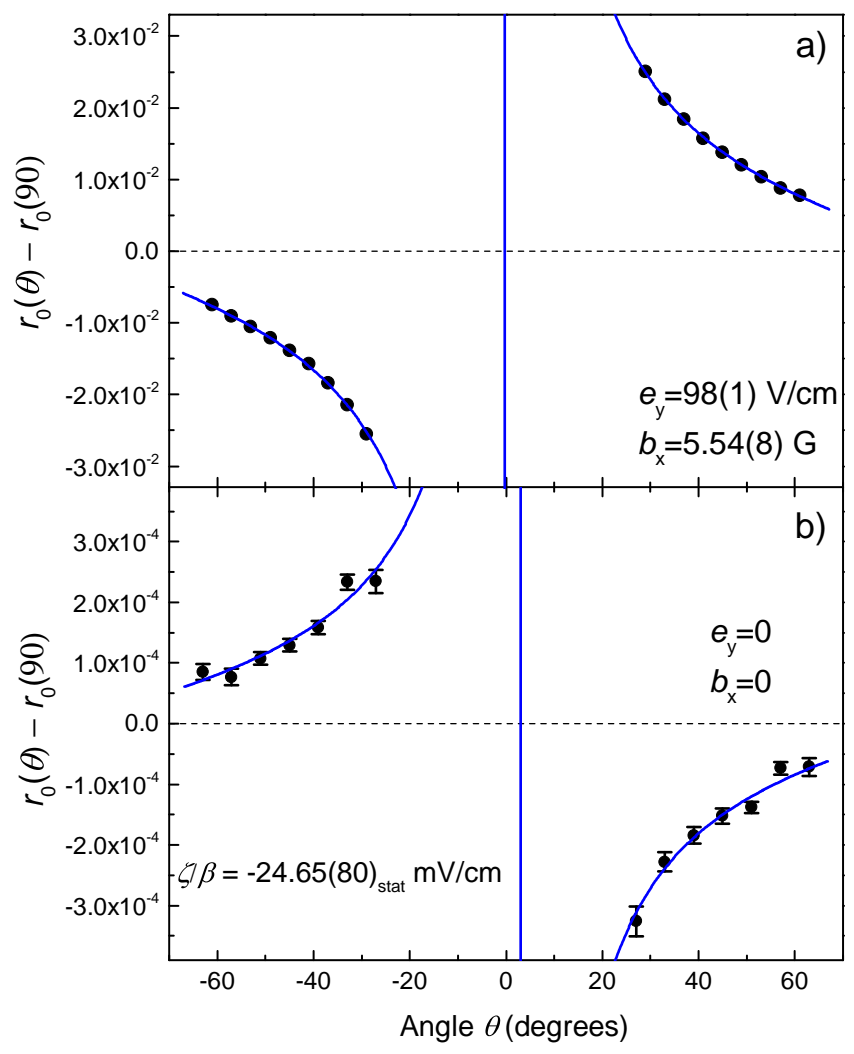

FIG. 10. (Color online) Harmonics ratio dependence on polarization angle, measured for ${ }^{174} \mathrm{Yb}$, with a) application of enhanced field imperfections $b_{x}$ and $e_{y}$ (which are both positive as is the $93 \mathrm{G}$ leading field $B_{z}$ ), resulting in a dominant contribution to $r_{0}$ from the term $\left.\left(b_{x} / B_{z}\right) e_{y} \cot \theta, \mathrm{b}\right)$ without applied imperfections to the atoms, thus primarily observing the PV-related term $(\zeta / \beta) \cot \theta$. The measured ratio $r_{0}(\pi / 2)$ is subtracted from data taken at other angles $\theta$, to eliminate background signal which does not depend on $\theta$. Error bars in a) are smaller than data points. The blue line in both plots is a fit to the data of the form $y=y_{0}+A \cot \left(\theta-\theta_{0}\right)$. Observation of the change in the harmonics ratio with $\theta$ in a) offers a confirmation for the sign definition for $\theta$. Furthermore, the $\theta$-dependence of the ratio in b) provides an unambiguous determination of the sign of $\zeta / \beta$. The data of b) also provide a measurement of $\zeta / \beta$, which is consistent with the final result of $-23.90(11) \mathrm{mV} / \mathrm{cm}$ for the ${ }^{174} \mathrm{Yb}$ isotope.

are were mentioned in [1. Here we give a more detailed account of these and provide the respective results in Table II

The PV isotopic comparison was carried out on the $m=0 \rightarrow m^{\prime}=0$ component of the $408 \mathrm{~nm}$ transition, since the systematics in this component are fewer compared to the $m=0 \rightarrow m^{\prime}= \pm 1$ transitions, as shown in Appendix A. However, to verify that the PV-effect is of opposite sign between the $0 \rightarrow 0$ and $0 \rightarrow \pm 1$ transitions, as expected by our model (see Appendix A), data were also taken on the latter components. Indeed, it was observed that the PV effect switches sign between the $0 \rightarrow 0$ and $0 \rightarrow \pm 1$ transitions. To check another expec- 
tation, that the $\mathrm{PV}$ effect on the $0 \rightarrow 0$ transition has a $\cot \theta$ dependence [see eq. (12)], the dependence of the harmonics ratio $r_{0}$ on the angle $\theta$ was investigated. This expectation was confirmed, as is shown in fig. 10 b. The resulting value for $\zeta / \beta$ from these data is consistent with the final measurement for the ${ }^{174} \mathrm{Yb}$, shown in the first line of Table II

Experiments were done using enhanced field imperfection in the interaction region. PV measurements on the $m=0 \rightarrow m^{\prime}=0$ were made with enlarged reversing fields $e_{y}^{r}, e_{z}^{r}$ or non-reversing field $e_{z}$. The respective results did not reveal unaccounted-for systematics dependent upon misalignment of the primary electric field, or a stray $e_{z}$ field, at the $\approx 2 \%$ level. (The effects of the large dc field $E_{d c}$ in the x-direction, as well as a stray field $e_{y}$, were also studied thoroughly-see sections IV B 3 and IV B 1.)

The isotopic comparison data were taken with the 408 $\mathrm{nm}$ laser frequency stabilized to the primary peak of the transition lineshape (see fig. 3b). Systematics related to the transition lineshape are not expected in the $\mathrm{Yb}$ apparatus. Such systematics were present in the Cs PV experiment 15], owing to the imperfect cancellation of the Stark-M1 interference in that work. Here this interference is suppressed to a much greater degree (see Appendix A. . To check this expectation, two different experiments were carried out. The first involved PVdata acquisition on the secondary peak of the $0 \rightarrow 0$ lineshape. The result for $\zeta / \beta$ was consistent with that obtained from measurements on the primary peak. The second experiment involved acquiring spectra of the 408 nm transition, such as that shown in fig. 3, and fitting to the complete lineshape (i.e. to all three lineshape components $0 \rightarrow 0, \pm 1$, just as it was done in the earlier $\mathrm{Yb}$ work [37, 38]). This method yielded a $\zeta / \beta$ value which is consistent with that obtained from the data solely on the $0 \rightarrow 0$ transition. The statistical sensitivity in this lineshape-fitting method was lower than that of the measurements with the laser stabilized to the peak of the $0 \rightarrow 0$ transition. This is primarily because the impact of laser frequency- noise on measurements done on the side of a peak, is greater than that for data taken at the top of a peak. The effective signal-to-noiseratio (SNR) in measuring $\zeta / \beta$ with the lineshape-fitting method was $0.06 \sqrt{\tau(s)},(\tau$ is the integration time), where as the SNR for PV data taken with the laser locked on the $0 \rightarrow 0$ transition was approximately 9 times greater, as discussed in the methods section of [1].

Further checks with the $\mathrm{Yb}$ apparatus were done to confirm a null result for the PV effect in particular cases. A PV effect should not be observed, for instance, when the excitation of atoms is done with circularly polarized light. In such a case, the light ellipticity [see (11)] $\phi= \pm \pi / 2$, and no PV-related contribution appears in the harmonics ratio $r_{0}$ 12. A null result was confirmed under such conditions, as shown in TableII. Another related experiment made use of the ${ }^{171} \mathrm{Yb}$ isotope. The ground state ${ }^{1} \mathrm{~S}_{0}$ in this nuclear-spin-isotope $(I=1 / 2)$, has a single hyperfine level with total angular momentum $F=$ $I=1 / 2$ ( electronic angular momentum $J=0$ ), where as the excited state ${ }^{3} \mathrm{D}_{1}\left(J^{\prime}=1\right)$ has two hyperfine levels with $F^{\prime}=1 / 2$ or $3 / 2$. With application of the typical $93 \mathrm{G}$ magnetic field, the Zeeman sublevels of the excited state are spectrally separated, however the ground state sublevels experience negligible splitting, since $J=0$. Exciting atoms with linearly polarized light to a particular $m_{F^{\prime}}$ sublevel through the $F=1 / 2 \rightarrow F^{\prime}=1 / 2$ transition (selection rules $\Delta m=0, \pm 1$ ), leads to contributions to the signal from both ground state $m_{F}= \pm 1$ levels. As the Stark-PV interference contributions for the two transitions $m_{F}= \pm 1 / 2 \rightarrow m_{F^{\prime}}$ are opposite, no PV observable is expected on the $F=1 / 2 \rightarrow F^{\prime}=1 / 2$ transition. A null measurement confirmed this and is presented in Table II

To obtain additional confidence that the detection of the PV effect if free of spurious apparatus contributions, measurements were done under drastically different conditions. For instance, we took data using a simply constructed set of electric field plates which replaced the elaborate set of electrodes shown in fig. 2. These measurements are shown in TableII. Another experiment was done with use of a travelling-wave field to excite the 408 $\mathrm{nm}$ transition, i.e. without the PBC. The result of the latter measurements is consistent with the final result for the ${ }^{174} \mathrm{Yb}$ isotope, with the $30 \%$ error being the consequence of poor statistical sensitivity due to the decreased $408 \mathrm{~nm}$ optical intensity.

Further information about the consistency of the actual isotopic comparison data can be obtained from the analysis of the combinations of Table I] The quantity $K_{1}$ is used to determine the $\mathrm{PV}$ effect; $K_{4}$ is nominally invariant under the $\theta$ - and $B$-reversals, and is used to make a precise $E_{d c}$ reversal during data acquisition. The combinations $K_{2}$ and $K_{3}$ are related to products of field imperfections (and $\zeta / \beta$ ) and are expected to be small compared to the measured PV effect. We show in fig. 11 data related to $K_{2}$ and $K_{3}$ coming from the actual PV run on the four $\mathrm{Yb}$ isotopes. Since data were taken at different electric fields, instead of $K_{i}$, the effective electric field $E_{i}=E_{0} K_{i} / 16(\mathrm{i}=2,3)$ is shown in fig. 11, that can be directly compared to the determined effective PV field $|\zeta / \beta| \approx 23.5 \mathrm{mV} / \mathrm{cm}$. The weighted mean of $E_{3}$ is $\approx 0.26 \%$ of $|\zeta / \beta|$ and consistent with zero within its $1 \sigma$ uncertainty, and the weighted mean of $E_{2}$ is $\approx 0.51 \%$ of $|\zeta / \beta|$ and consistent with zero within $2 \sigma$.

\section{RESULTS AND ANALYSIS}

In this section we present the results of the PV-isotopic comparison run that took place within a 2.5 month period, from $11 / 2017$ to $01 / 2018$. We compare the observed isotopic variation of the $\mathrm{PV}$ effect with the prediction of the SM for this variation. In addition, we present an analysis of these measurements, that is used to constrain electron-nucleon interactions due to the presence of an 
TABLE II. Results of auxiliary experiments

\begin{tabular}{cccc}
\hline \hline Isotope mass number & Transition & Type of experiment & $\zeta / \beta(\mathrm{mV} / \mathrm{cm})$ \\
\hline 174 & $m=0 \rightarrow m^{\prime}=0$ & Actual isotopic comparison data & $-23.89(11)$ \\
174 & $m=0 \rightarrow m^{\prime}= \pm 1$ & $\ldots$ & $23.30(26)$ \\
174 & $m=0 \rightarrow m^{\prime}=0$ & Measurement of $r_{0}$ vs. $\theta$ b & $-24.65(80)$ \\
174 & $m=0 \rightarrow m^{\prime}=0$ & Enhanced $e_{y}^{r} / E_{0}=-0.03$ & $-24.30(48)$ \\
174 & $m=0 \rightarrow m^{\prime}=0$ & Enhanced $e_{y}^{r} / E_{0}=0.03$ & $-23.93(40)$ \\
174 & $m=0 \rightarrow m^{\prime}=0$ & Enhanced $e_{z}^{r} / E_{0}=-0.029$ & $-23.98(57)$ \\
174 & $m=0 \rightarrow m^{\prime}=0$ & Enhanced $e_{z}^{r} / E_{0}=0.029$ & $-23.76(57)$ \\
174 & $m=0 \rightarrow m^{\prime}=0$ & Enhanced $e_{z} / E_{0}=-0.076$ & $-24.67(57)$ \\
174 & $m=0 \rightarrow m^{\prime}=0$ & Enhanced $e_{z} / E_{0}=0.076$ & $-23.83(57)$ \\
174 & $m=0 \rightarrow m^{\prime}=0$ & Measurement on secondary transition peak & $-24.14(44)$ \\
174 & $m=0 \rightarrow m^{\prime}=0, \pm 1$ & Lineshape fitting & $-21(4)$ \\
171 & $F=1 / 2 \rightarrow F^{\prime}=1 / 2$ & . & $-0.59(57)$ \\
174 & $m=0 \rightarrow m^{\prime}=0$ & 408 nm excitation using circularly-polarized light & $-0.2(12)$ \\
174 & $m=0 \rightarrow m^{\prime}=0$ & Measurement with different field plates & $-25.2(12)$ \\
174 & $m=0 \rightarrow m^{\prime}=0$ & Measurement without PBC & $-26(7)$ \\
\hline \hline
\end{tabular}

a The PV-mimicking terms $e_{y}^{r}\left(e_{z} / E_{0}\right)$ and $e_{z}^{r}\left(e_{y} / E_{0}\right)$ were not compensated prior to the measurement (see Appendix A and eq. A24).

b See fig. 10

c Done without the high degree of $408 \mathrm{~nm}$ polarization control implemented in the isotopic comparison runs.

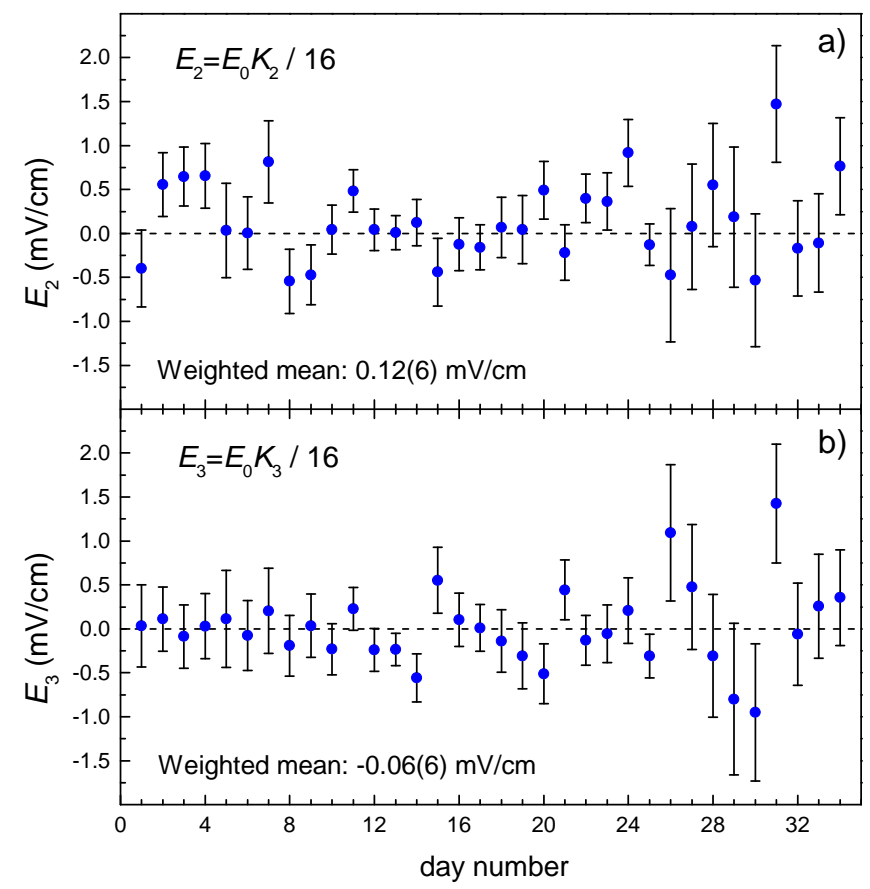

FIG. 11. (Color online) Effective electric field corresponding to the combination a) $K_{2}$ and b) $K_{3}$ (see table I). The data come from the isotopic comparison PV-run.

extra $Z^{\prime}$ boson.

\section{A. Results of the PV-measurements}

The results presented here are from data acquired on a chain of four $\mathrm{Yb}$ nuclear-spin-zero isotopes, with mass numbers $\mathrm{A}=170,172,174,176$, and abundances $3.1 \%$,
$21.9 \%, 31.8 \%, 12.9 \%$, respectively. Measurements were made on the $m=0 \rightarrow m^{\prime}=0$ component of the $408 \mathrm{~nm}$ transition, in 34 days, for a total of $420 \mathrm{hr}$ of integration with $\approx 62 \%$ duty cycle. A typical routine in the experiment involved loading $\mathrm{Yb}$ metal into the oven, studying PV-mimicking systematics, followed by a five day measurement run, of an average $12 \mathrm{hr}$ long daily data-taking time each day.

The data-acquisition routine was divided in $\approx 30 \mathrm{~min}$ long blocks (PV runs). A PV run consisted of a set of 200 determinations of the harmonics ratio $r_{0}$, made under all combinations of polarities for the parameters $E, \theta$, $B$ and $E_{d c}$. The primary experimental reversal, which is a parity-reversal, was that of the electric field, which was reversed at a rate of $19.9 \mathrm{~Hz}$. The second parity reversal is a $\pm \pi / 2$ rotation in $\theta$, which occurred at 0.12 $\mathrm{Hz}$. The primary magnetic field $B$ was reversed at 0.06 $\mathrm{Hz}$ and the field $E_{d c}$ at and $0.03 \mathrm{~Hz}$. The amplitude of the applied ac-field was $E_{0} \approx 812$ or $1218 \mathrm{~V} / \mathrm{cm}$ (1218 or $1624 \mathrm{~V} / \mathrm{cm}$ with ${ }^{170} \mathrm{Yb}$ ). The polarization angle values were $\theta_{ \pm} \approx \pm \pi / 4$. A total of $884 \mathrm{PV}$-runs were done, with the number of runs per isotope varying, depending on its respective abundance. Measurements were alternated among the four spin-zero $\mathrm{Yb}$ isotopes, to minimize the impact of potential apparatus drifts.

The measured $\zeta / \beta$ value in each of the four isotopes, is shown in Table III. Our quoted result is the weighted mean of the set of measurements (PV runs) made on the particular isotope. The statistical uncertainty given in Table III is the standard error of the respective weighted mean. The systematic uncertainty of $0.06 \mathrm{mV} / \mathrm{cm}$ is the same for all isotopes. The main sources of this uncertainty were discussed in section [V] and their respective contributions are presented in Table IV.

Statistical consistency of the obtained sets of PV data is indicated by the resulting $\chi^{2}$ value for each isotope, 

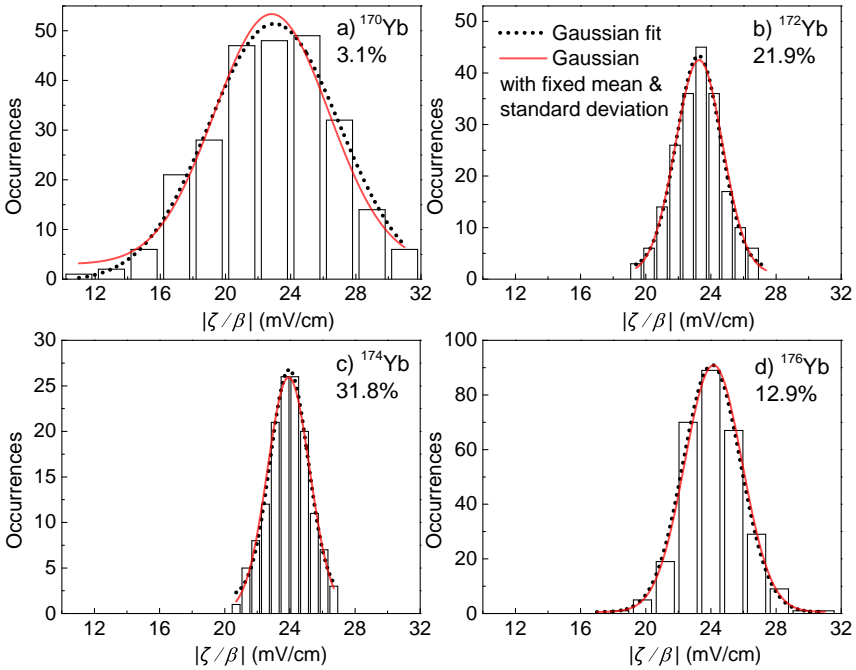

FIG. 12. (Color online) Occurrences of $|\zeta / \beta|$ values observed in 30-min long $\mathrm{PV}$-runs, shown for each on the four $\mathrm{Yb}$ isotopes, whose respective abundances are given in the plots. The distribution of measurements is well-approximated by a Gaussian fit (dotted-black line), indicating that the PVdata are randomly distributed. Also shown in these plots is a solid-red line representing a Gaussian with center given by the mean value from Table III and standard deviation which is the standard error of this mean multiplied by $\sqrt{N}$, where $N$ is the number of data points (PV runs) given in Table III

as well as by the probability value associated with the respective set. Consistency of the data is also supported by the frequency count plots of fig. 12 in which a random distribution of the measurements is observed.

The parameter $\zeta / \beta$ for ${ }^{174} \mathrm{Yb}$ was reported in [37, 38 as $39(4)_{\text {stat }}(5)_{\text {syst }} \mathrm{mV} / \mathrm{cm}$. This magnitude is significantly larger than that of the present determination for the same isotope, of $23.89(11)_{\text {stat }}(0.06)_{\text {syst }}$. The lack of ability to investigate systematics in the apparatus used in [37, 38, makes it challenging to trace the source of the discrepancy. It is possible that due to the much lower sensitivity of the old apparatus, systematic effects were underestimated.

The statistical error of the $30 \mathrm{~min}$ long $\mathrm{PV}$ run varied between $5 \%$ for the highest-abundance isotope $\left({ }^{174} \mathrm{Yb}\right)$ to $16 \%$ for the lowest-abundance $\left({ }^{170} \mathrm{Yb}\right)$. The SNR in detection of the PV-effect was $0.55 \sqrt{\tau}$ ( $\tau$ is the integration time in s) for the highest-abundance isotope. The observed SNR levels in the PV-data acquisition are roughly consistent with shot-noise-limited detection of the 408 $\mathrm{nm}$ excitations in the atomic beam. To illustrate this, we compute the SNR for detection of the Stark-PV interference signal $S_{S t-P V}=c_{1} n \zeta \beta E_{0}$ in the presence of the Stark-induced signal $S_{S t}=c_{2} n \beta^{2} E_{0}^{2}$. The parameter $n$ is the atomic beam density, and $c_{1}, c_{2}$ are constants. The noise in detection of $S_{S t-P V}$ has three contributions: background $(B G)$ noise (independent of $S_{S t}$ ), technical noise $T \cdot S_{S t}$ (i.e. proportional to the signal, with $T$ a constant), and shot-noise $S \sqrt{S_{S t}}$, where $S$ is a constant.

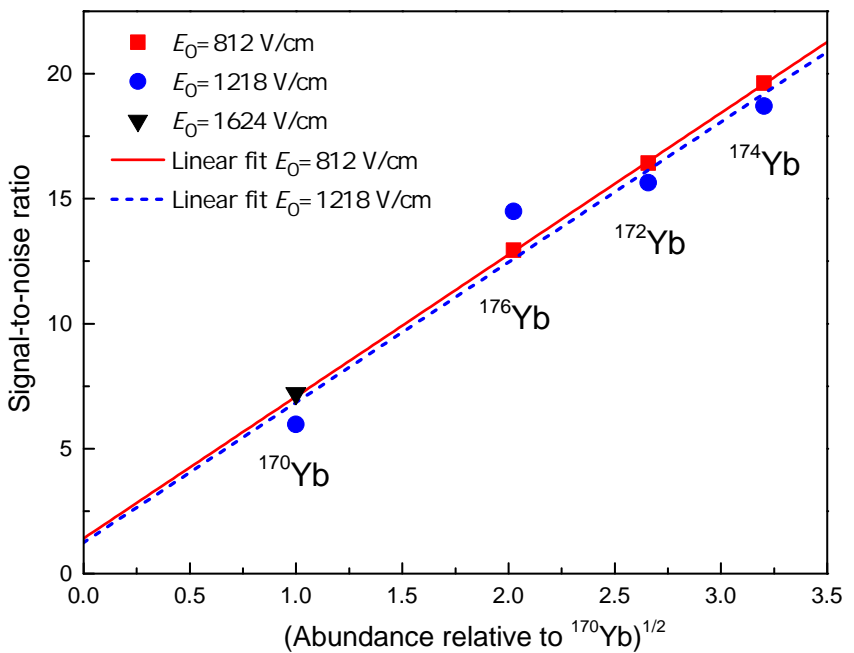

FIG. 13. (Color online) Obtained SNR in measurements of $\zeta / \beta$ in a 30 min long $\mathrm{PV}$-run, plotted against the square root of the isotopic abundance (or equivalently, the effective atomic beam density). Values are shown for all three different electric fields for which data were taken. Each value in the plot is computed by multiplying the obtained standard error of the weighted mean value $\zeta / \beta$ in the corresponding set of data by $\sqrt{N}$, where $N$ is the number of measurements in the set.

With quadrature addition of these contributions, we obtain for the SNR:

$$
\begin{aligned}
S N R= & \frac{S_{S t-P V}}{\text { noise }} \\
& =\frac{c_{1} n \zeta \beta E_{0}}{\sqrt{B G^{2}+S^{2} c_{2} n \beta^{2} E_{0}^{2}+T^{2} c_{2}^{2} n^{2} \beta^{4} E_{0}^{4}}} .
\end{aligned}
$$

When shot-noise is the dominant noise-source $\left(S^{2} c_{1} n \beta^{2} E_{0}^{2} \gg B G^{2}, \quad T^{2} c_{2}^{2} n^{2} \beta^{4} E_{0}^{4}\right)$, the SNR is given by:

$$
S N R \approx \frac{c_{1} \zeta}{S \sqrt{c_{2}}} \sqrt{n} .
$$

We see from 29 that the shot-noise-limited SNR does not depend on the electric field $E_{0}$, and that it scales linearly with $\sqrt{n}$. Fig. 13 shows the observed SNR of a typical PV run per isotope and per value of $E_{0}$. The SNR grows approximately as the square root of the isotope abundance, and it has little dependence on the electric field. These observations indicate that the detection of the PV effect approaches the shot-noise limit. Apparatus and measurement method-improvements that resulted in this sensitivity enhancement, relative to that in the $1^{\text {st }}$ generation experiment [37, 38, are discussed in 1].

\section{B. Isotopic variation of the PV-effect and comparison with SM prediction}

The uncertainty in the present measurements is lowenough to allow for observation of the isotopic variation 
TABLE III. Results of PV measurements

\begin{tabular}{|c|c|c|c|c|c|}
\hline Isotope mass number & Abundance (\%) & Number of PV runs & $\zeta / \beta(\mathrm{mV} / \mathrm{cm})$ & $\chi^{2} /$ d.o.f. & $p$-value ${ }^{a}$ \\
\hline 170 & 3.1 & 254 & $-22.81(22)_{\text {stat }}(0.06)_{\text {syst }}$ & 1.09 & 0.16 \\
\hline 172 & 21.9 & 199 & $-23.24(10)_{\text {stat }}(0.06)_{\text {syst }}$ & 0.92 & 0.77 \\
\hline 174 & 31.8 & 140 & $-23.89(11)_{\text {stat }}(0.06)_{\text {syst }}$ & 0.99 & 0.53 \\
\hline 176 & 12.9 & 291 & $-24.12(10)_{\text {stat }}(0.06)_{\text {syst }}$ & 1.02 & 0.41 \\
\hline
\end{tabular}

${ }^{\text {a }}$ Probability that a repeated experiment would yield a $\chi^{2}$ value greater than the observed one.

TABLE IV. Main systematic uncertainties in the PV measurements [1].

\begin{tabular}{lc}
\hline \hline Contribution & Uncertainty $(\%)$ \\
\hline Harmonics ratio calibration & 0.22 \\
Polarization angle & 0.1 \\
High-voltage measurements & 0.06 \\
Transition saturation correction & $0.05{ }^{\natural}$ \\
Field-plate spacing & 0.04 \\
Stray fields \& field misalignments & 0.02 \\
Photo-detector response calibration & 0.02 \\
\hline \hline
\end{tabular}

${ }^{a} 0.09$ for ${ }^{170} \mathrm{Yb}$. The error is larger because part of the data for this low-abundance isotope were taken at a higher electric field.

of the PV effect, and a comparison of this variation with the related prediction of the SM. The effect predicted by the SM scales as the weak charge of the nucleus $Q_{W}$, which to lowest order in the SM is given by [2]:

$$
Q_{W}=-N+Z\left(1-4 \sin ^{2} \theta_{W}\right),
$$

where $Z, N$ are the number of protons and neutrons in the nucleus and $\theta_{W} \approx 29.2^{\circ}$ is the weak-mixing angle [52]. A more accurate expression for $Q_{W}$ [52] is obtained with inclusion of radiative corrections:

$$
Q_{W} \approx-0.989 N+0.071 Z \text {. }
$$

This expression should be accurate at the $0.1 \%$ level. For the mean neutron number $N=103$ of the isotopes measured in this experiment, and $Z=70$, the corresponding weak charge $Q_{W}=-96.88$, with a proton contribution $Q_{p}=4.98$. About $95 \%$ of the $\mathrm{Yb}$ nucleus weak charge is carried by neutrons. The expected by the SM fractional variation in $Q_{W}$ per neutron, around $\mathrm{N}=103$ is:

$$
V_{S M}=\frac{1}{Q_{W}} \frac{d Q_{W}}{d N} \approx 1 \% .
$$

A clear variation of the measured PV effect is seen in fig. 14, in which the determined $(-\zeta / \beta)$ values for the different isotopes vs. the neutron number are shown. The measured fractional variation in the PV effect per neutron, around $\mathrm{N}=103$, is:

$$
V_{\text {exp }}=\frac{\text { slope }}{(-\zeta / \beta)_{N=103}}
$$

From the parameters of the fit to the data of fig. 14, we obtain $V_{e x p}=0.96(15) \%$. In addition, the y-intercept

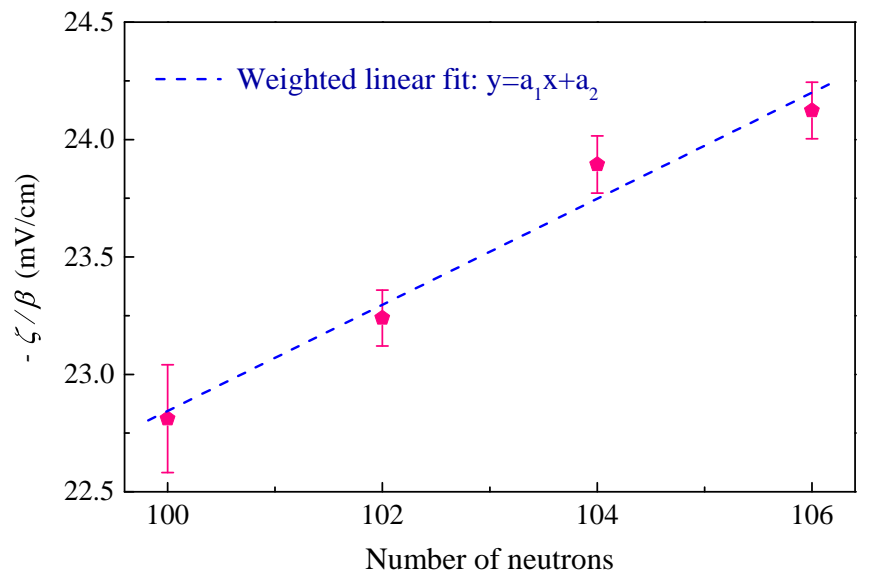

FIG. 14. (Color online) Isotopic variation of the parameter $-\zeta / \beta$. The error bars shown come from quadrature addition of the statistical and systematic errors and the weight assigned to each point to perform the weighted linear fit to these data is the inverse square of the respective error bar. The parameters of the linear fit are: $a_{1}=0.226 \pm 0.035 \mathrm{mV} / \mathrm{cm}$ and $a_{2}=0.3 \pm 3.6 \mathrm{mV} / \mathrm{cm}$. The reduced $\chi^{2}$ value of the fit is 1.04 . Figure adapted from [1].

of the fitted line is consistent with the expected from the SM model contribution due to protons, estimated to be $\left(Q_{p} / Q_{W}\right) \cdot(-\zeta / \beta)_{N=103} \approx-1.2 \mathrm{mV} / \mathrm{cm}$ with the value $(-\zeta / \beta)_{N=103}=23.52 \mathrm{mV} / \mathrm{cm}$ obtained from the fit parameters, and $Q_{W}=-96.88$ for $\mathrm{N}=103$. The small size of the y-intercept is consistent with expectation that the PV effect is mainly due to the neutrons.

The measured variation of the PV effect $V_{\text {exp }}$ agrees well with the SM expectation $V_{S M}$, thus offering a direct confirmation of the $Q_{W}$ dependence on neutrons.

In determining the variation $V_{\text {exp }}$, the effects of the neutron skin and its variation among the four isotopes measured were neglected. This is reasonable, as the estimated fractional change in the PV effect between the two extreme isotopes ${ }^{170} \mathrm{Yb}$ and ${ }^{176} \mathrm{Yb}$, due to the variation in the neutron skin between these, is only about $0.1 \%$ [23, 24]. This variation is much smaller than the observed change of $\approx 5.7 \%$ between the two extreme isotopes.

The most precise determinations of a nuclear weak charge were made in ${ }^{133} \mathrm{Cs}\left(Q_{W}=-72.58(43)\right.$ [17]), ${ }^{205} \mathrm{Tl}\left(Q_{W}=-113(3)[53)\right.$, and ${ }^{208} \mathrm{~Pb}\left(Q_{W}=-114(9)\right.$ through a combination of measurements of the PV ef- 
fect with atomic calculations. These determinations combined, have provided a test of the SM regarding the dependence of the weak charge on neutrons and protons. However, taking an agnostic approach, one may question if there is direct evidence of the weak charge being dominated by neutrons. Indeed, we can plot the value of the weak charge inferred from $\mathrm{Cs}, \mathrm{Tl}$ and $\mathrm{Pb}$ experiments, and as a function of the number of neutrons (fig. 15). The dependence is well fit with a linear function with the slope close to the expected value of $\approx-1$. This fit, however, does not account for correlations in the number of protons and neutrons. To account for such correlations, one can consider instead a weighted fit to the data points of fig. 15, of the form:

$$
Q_{W}=A \cdot Z+B \cdot N
$$

The poor ability to reliably determine the parameters $A$ and $B$ from such a fit is illustrated in fig. 16 , which shows the distribution of the weighted sum of squares (wss) of the form:

$$
w s s(A, B)=\sum_{i} \frac{\left(A \cdot Z_{i}+B \cdot N_{i}-Q_{W, i}\right)^{2}}{\sigma_{i}^{2}},
$$

where $Q_{W, i}$ refer to the data points of fig. 15 and $\sigma_{i}$ are the respective errors. One can infer from fig. 16 that a least squares fit to (34) can not provide a reliable estimate for the parameters $A$ and $B$ independently, but rather on the linear combination of $A$ and $B$. Therefore, one can claim that the earlier experiments have not provided a model-independent way of showing that the weak charge is dominated by neutrons and is linear in the number of neutrons, the result we have been able to derive from the isotopic comparison in $\mathrm{Yb}$. To illustrate that the present experiment achieved that, we express the PVrelated parameter $\zeta / \beta$ as $\zeta / \beta=k_{a t} Q_{W}$, where $k_{a t}$ is a factor which would need to be calculated accurately to extract $Q_{W}$ from the experiment (see related discussion in section I). The quantity $\zeta / \beta$ can be further expressed as:

$$
\zeta / \beta=0.2428\left(A_{\text {exp }} \cdot Z+B_{\text {exp }} \cdot N\right) \mathrm{mV} / \mathrm{cm},
$$

where $k_{a t}=0.2428 \mathrm{mV} / \mathrm{cm}$ was computed using the value $Q_{W}=-96.88$ for $\mathrm{N}=103$ [see eq. [31)]. This value corresponds to $(\zeta / \beta)_{N=103}=-23.52 \mathrm{mV} / \mathrm{cm}$, which is extracted from the fit of fig. 14. With use of the results of the same fit, we determine the parameters of eq. (36): $A_{\text {exp }}=-0.01(21), B_{\exp }=-0.93(14)$. These values are in agreement with the expected by the SM values [eq. (31)]: $A_{S M}=0.071$ and $B_{S M}=-0.989$.

\section{Constraints on $Z^{\prime}$ bosons}

The results of the isotopic comparison can be used to place constrain PV couplings between electrons and nucleons that are mediated by an extra vector boson $Z^{\prime}$.

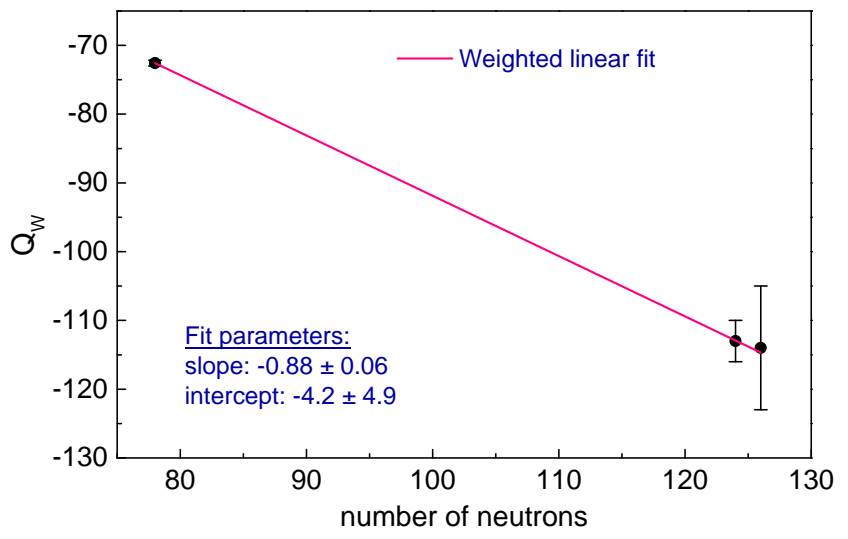

FIG. 15. (Color online) Nuclear weak charge plotted against number of neutrons. The data points come from the most precise weak charge determinations, made in ${ }^{133} \mathrm{Cs}(Z=55$, $N=78),{ }^{205} \mathrm{Tl}(Z=81, N=124)$, and ${ }^{208} \mathrm{~Pb}(Z=82, N=126)$. The weight assigned to each point in order to perform a weighted linear fit to these data, is the inverse square of the corresponding error bar shown in the plot. Errors in the fit parameters are the $1 \sigma$ errors.

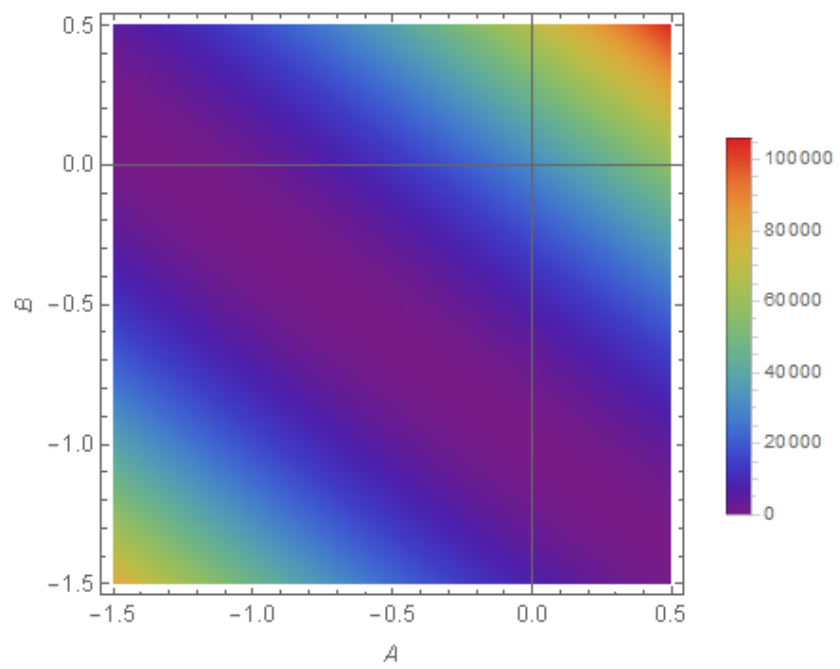

FIG. 16. Distribution of the quantity wss of (35) using the data of fig. 15 Violet color indicates the region of smaller values for wss and red indicates the region with the largest values within the plotted parameter space. The violet region is a strip extending to infinity.

A number of searches for light vector bosons of mass $m_{Z^{\prime}}>100 \mathrm{keV}$, as well as searches for interactions of SM matter with dark-matter bosons and dark-energy fields have been reported (see, for example, review 4 and references therein). Constraints on $Z^{\prime}$-mediated interactions were placed from torsion-pendulum [54, 55] and atomic-magnetometry [56] experiments, as well as from atomic calculations [25] that employed analyses of results of the Cs PV experiment [15]. These constraints are on combinations of electron-proton and electron-neutron PV interactions. The isotopic-comparison measurements 
allow for extraction of the proton contribution to the PV effect. This separation of the electron-proton PV coupling is used to provide individual constraints on an additional electron-proton PV interaction due to $Z^{\prime}$ exchange. These new constraints can be combined with existing upper bounds on the sum of electron-proton and electron-neutron couplings, to place a separate limit on electron-neutron interactions.

The interactions considered here arise in the presence of a $Z^{\prime}$ boson which does not kinetically mix with the $\mathrm{Z}$ boson of the SM. The following $Z^{\prime}$-mediated interaction is assumed between the electron and nucleons:

$$
\mathcal{L}=Z_{\mu}^{\prime} \sum_{f=e, p, n} \bar{f} \gamma^{\mu}\left(g_{f}^{V}+\gamma_{5} g_{f}^{A}\right) f
$$

where $Z_{\mu}^{\prime}$ and $f$ are respectively the boson and fermion amplitudes, and $\gamma^{\mu}$ are Dirac matrices.

The present isotopic comparison data provide an estimate for the proton contribution to the PV parameter $\zeta / \beta$. This estimate is used in combination with the atomic calculations of 25] to place the upper bound on the axial electron-vector proton coupling $g_{e}^{A} g_{p}^{V}$. The atomic PV calculations reported in [25] assume a finite mass for the $Z^{\prime}$ boson. Therefore the corresponding couplings and bounds of these are defined for any mass $m_{Z^{\prime}}$, and not only in the limit of a mass which is large on the atomic scale. The bound obtained on $g_{e}^{A} g_{p}^{V}$ is combined with a previous bound on an effective electron-nucleon coupling $g_{e}^{A} g_{N}^{V}$ (see analysis in [25]) to constrain the axial electron-vector neutron coupling $g_{e}^{A} g_{n}^{V}$. A detailed account of the analysis to derive bounds on $g_{e}^{A} g_{p}^{V}$ and $g_{e}^{A} g_{n}^{V}$ is given in [1], and here we only provide its main results. We show in fig. 17 the constraints derived on the $Z^{\prime}$-mediated electron-proton and electron-neutron couplings. In Table $\mathrm{V}]$ we present all the asymptotic values for the couplings $g_{e}^{A} g_{p}^{V}$ and $g_{e}^{A} g_{n}^{V}$ in the limits of low mass and high mass for $Z^{\prime}$.

\section{CONCLUSIONS AND OUTLOOK}

We discussed in detail the experimental principle used to make improved measurements of the PV effect in a chain of four $\mathrm{Yb}$ isotopes. We described the $2^{n d_{-}}$ generation atomic beam apparatus, which offers enhanced sensitivity in the detection of the PV effect, thus enabling better characterization of systematic effects in these measurements. We gave a detailed account of the studies of these systematic effects, in relation to the isotopic-comparison experiment.

The results of the PV measurements presented here offer the first direct observation of isotopic dependence in atomic PV. The measured variation in the PV effect, of $0.96(15) \%$ per neutron, is in agreement with the expectation based on the electroweak theory, of $\approx 1 \%$ per neutron. Our result is consistent with the notion of the magnitude of the neutron weak charge being close to unity

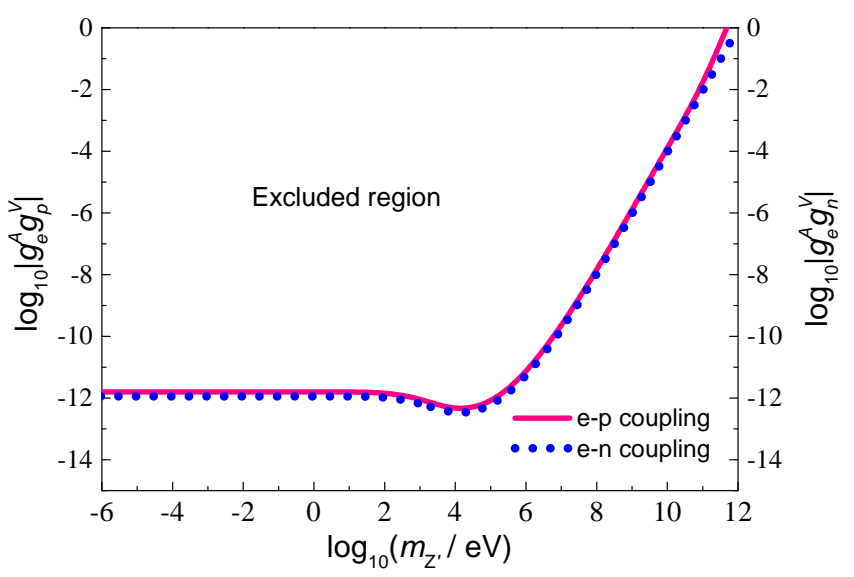

FIG. 17. (Color online) Constraints on PV electron-nucleon interactions, mediated by an extra $Z^{\prime}$ boson. The limit on the axial electron-vector proton interaction $g_{e}^{A} g_{p}^{V}$, represented by the pink line, is derived by analysis of the present PV results combined with previous atomic PV calculations 25]. The limit on the axial electron-vector neutron interaction $g_{e}^{A} g_{n}^{V}$, represented by the blue dotted line, comes from combination of the obtained bound on $g_{e}^{A} g_{p}^{V}$ with a previous result on an effective electron-nucleon coupling. The latter coupling was derived in [25] through analysis of the results of [15]. Both bounds are shown at the $67 \%$ confidence level. The low-mass and high-mass asymptotic limits for these bounds are given in Table $\mathrm{V}$. Figure adapted from [1].

(Eq. (30) ) and the weak charge of the nucleus to be additive over the neutrons.

The isotopic-comparison method allowed the extraction of the proton contribution to the PV effect. This contribution has enabled analysis that provided constraints on axial electron-vector proton interactions, mediated by a light boson $Z^{\prime}$. These new constraints were combined with existing constraints on the sum of electron-proton and electron-neutron couplings, to provide separate constraints on the latter.

The attained single-isotope uncertainty is $\approx 0.5 \%$ for three of the $\mathrm{Yb}$ isotopes measured. The present sensitivity level is a benchmark for the newly-built apparatus. Many avenues to enhance sensitivity have been identified and are currently being explored. These include an upgrade of the PBC cavity optics for a greater circulating power level, an optimization in the atomic beam flux, potentially involving laser cooling of the transverse velocity distribution of atoms.

A tenfold sensitivity enhancement should allow a measurement of the variation of neutron distributions in the $\mathrm{Yb}$ nucleus with use of the isotopic comparison method 22, 24. A tenfold sensitivity increase is also expected to be sufficient for an anapole moment measurement. The nuclear-spin-dependent PV amplitude, which is active for isotopes with nuclear spin $\left({ }^{171} \mathrm{Yb}, I=1 / 2,{ }^{173} \mathrm{Yb}\right.$, $I=5 / 2)$, contributes by $\approx 0.1 \%$ to the overall $\mathrm{PV}$ effect [20, 36, 57, 58 but this contribution depends on the particular hyperfine level. PV measurements on different hy- 
TABLE V. Upper bounds on electron-proton and electron-neutron interactions mediated by a vector boson $Z^{\prime}$ of mass $m_{Z^{\prime}}$. These limits are derived through analysis of the results of different experiments or combinations of these. The large-mass limits $g_{e}^{A} g_{p}^{V} / m_{Z^{\prime}}^{2}$ and $g_{e}^{A} g_{n}^{V} / m_{Z^{\prime}}^{2}$ are valid for $m_{Z^{\prime}}>1 \mathrm{GeV}$ and the low-mass limits $g_{e}^{A} g_{p}^{V}$ and $g_{e}^{A} g_{n}^{V}$ for $m_{Z^{\prime}}<100 \mathrm{eV}$ (see table I in [25]). Table reproduced from supplementary material of [1].

\begin{tabular}{|c|c|c|c|c|}
\hline & $\begin{array}{c}g_{e}^{A} g_{p}^{V} / m_{Z}^{2}(\mathrm{GeV})^{-2} \\
(\text { large-mass limit })\end{array}$ & $\begin{array}{c}g_{e}^{A} g_{n}^{V} / m_{Z}^{2}(\mathrm{GeV})^{-2} \\
(\text { large-mass limit })\end{array}$ & $\begin{array}{c}g_{e}^{A} g_{p}^{V} \\
\text { (low-mass limit) }\end{array}$ & $\begin{array}{c}g_{e}^{A} g_{n}^{V} \\
(\text { low-mass limit })\end{array}$ \\
\hline \multicolumn{5}{|l|}{ Experiment } \\
\hline Yb PV & $(3.7 \pm 9) \cdot 10^{-7}$ & $\cdots$ & $(4.5 \pm 11) \cdot 10^{-13}$ & $\cdots$ \\
\hline $\mathrm{Yb} \&$ Cs PV & & $(-2.9 \pm 6.4) \cdot 10^{-7}$ & $\ldots$ & $(-3.5 \pm 7.9) \cdot 10^{-13}$ \\
\hline Q-weak & $(-4.5 \pm 18.6) \cdot 10^{-9}$ & $\ldots$ & $\ldots$ & .. \\
\hline Q-weak \& Cs PV & $\cdots$ & $(-3.1 \pm 2.6) \cdot 10^{-8}$ & $\cdots$ & $\ldots$ \\
\hline
\end{tabular}

perfine levels in the same fermionic isotope are therefore required to probe an anapole. For instance, an anapole extraction can be done by measuring the difference in the PV amplitudes between the $F=1 / 2 \rightarrow F^{\prime}=1 / 2$ and $F=1 / 2 \rightarrow F^{\prime}=3 / 2$ transitions in ${ }^{171} \mathrm{Yb}$, or between the $F=5 / 2 \rightarrow F^{\prime}=3 / 2$ and $F=5 / 2 \rightarrow F^{\prime}=7 / 2$ transitions in ${ }^{173} \mathrm{Yb}$. Optical pumping to an extreme magnetic sublevel of the $\mathrm{Yb}$ ground state, will improve statistical sensitivity and simplify the analysis of systematics. This pumping is necessary, in order to obtain a $\mathrm{PV}$ observable on the $F=1 / 2 \rightarrow F^{\prime}=1 / 2$ component of ${ }^{171} \mathrm{Yb}$ (see discussion in section IV C 2 .

An increase in the experimental sensitivity must be accompanied by improved understanding and control of systematic effects. With consideration to improved isotopic comparison measurements on a chain of nuclearspin-zero isotopes, systematic effects should not pose substantial difficulties. This is because the energy level structure is identical for the different isotopes, and since the influence of such spurious effects on measurements made on the $\Delta m=0$ transition is only moderate. The various calibrations applied to the data, as well as the associated uncertainties, are also largely independent of isotope measured.

Greater attention to systematics is required in the studies of spin-dependent PV. It is possible that some effects could contribute differently among the different hyperfine transitions, and affect the results of hyperfine comparison. A substantial amount of related studies was done in the Cs experiment [15], which (similarly to the present work) employed the Stark-PV interference method and was done with an atomic beam, with the use of a standing-wave field to excite atoms. Systematic contributions influencing the hyperfine comparison in that work came from the presence of a $M 1$ transition amplitude, which, although suppressed due to the use of a standing-wave, was allowed by the geometry of applied fields. In the $\mathrm{Yb}$ experiment, in addition to the suppression provided by the $\mathrm{PBC}$, the experimental geometry is such that the Stark and $M 1$ amplitudes are out of phase for the $\Delta m=0$ transition that we employ, and therefore they do not interfere. In addition to $M 1-$ related systematics, the effects of an electric-quadrupole (E2) transition between the ${ }^{1} \mathrm{~S}_{0}$ and ${ }^{3} \mathrm{D}_{1}$ states need to be considered. The $E 2$ transition is weakly allowed in the nonzero-spin isotopes due to hyperfine interactioninduced mixing between the ${ }^{3} \mathrm{D}_{1}$ and ${ }^{3} \mathrm{D}_{2}$ states. A detailed evaluation of the $E 2$ amplitudes in the ${ }^{1} \mathrm{~S}_{0} \rightarrow{ }^{3} \mathrm{D}_{1}$ transition was reported in [59. Fortunately, the same mechanisms employed to suppress the Stark and $M 1$ effects in PV measurements (experimental field geometry, excitation with counter-propagating light beams, selection of $\Delta m=0$ transitions), are expected to provide adequate suppression of Stark- $E 2$ signal contributions in the nonzero-spin isotopes. Modeling of systematics in the these isotopes, just as it was done for the studies presented here, shows that parasitic contributions to the true PV signal should be similar to those in the spin-zero isotopes. While the analysis indicates that it should be possible to control systematics in the measurements of the nuclear-spin-dependent PV, we expect that during the course of the $\mathrm{Yb} \mathrm{PV}$ program, studies of systematics will require most of our attention.

\section{ACKNOWLEDGEMENTS}

We are gratetful to M. Safronova, M. Kozlov, S. Porsev, M. Zolotorev, A. Viatkina, Y. Stadnik, L. Bougas and N. Leefer for fruitful discussions. VF thanks Gutenberg Fellowship and Australian Research Council. AF is supported by the Carl Zeiss Graduate Fellowship.

[1] D. Antypas, A. Fabricant, J. E. Stalnaker, K. Tsigutkin, V. V. Flambaum, and D. Budker, Nat. Phys. 15, 120 
[2] J. S. Ginges and V. V. Flambaum, Phys. Rep. 397, 63 (2004)

[3] B. Roberts, V. Dzuba, and V. Flambaum, Ann. Rev. Nucl. Part. Sci. 65, 63 (2015)

[4] M. S. Safronova, D. Budker, D. DeMille, D. F. J. Kimball, A. Derevianko, and C. W. Clark, Rev. Mod. Phys. 90, 025008 (2018)

[5] M. A. Bouchiat and C. C. Bouchiat, Phys. Lett. B 48, $111(1974)$

[6] Y. B. Zel' Dovich, J. Exptl. Theoret. Phys. (U.S.S.R.) 36, 964 (1959).

[7] L. M. Barkov and M. S. Zolotorev, JETP Lett. 27, 357 (1978).

[8] R. Conti, P. Bucksbaum, S. Chu, E. Commins, and L. Hunter, Phys. Rev. Lett. 42, 343 (1979).

[9] M. A. Bouchiat, J. Guena, L. Pottier, and L. Hunter, Phys. Lett. B 117, 358 (1982)

[10] M. J. D. MacPherson, K. P. Zetie, R. B. Warrington, D. N. Stacey, and J. P. Hoare, Phys. Rev. Lett. 67, 2784 (1991)

[11] D. M. Meekhof, P. Vetter, P. K. Majumder, S. K. Lamoreaux, and E. N. Fortson, Phys. Rev. Lett. 71, 3442 (1993)

[12] S. J. Phipp, N. H. Edwards, E. G. Baird, and S. Nakayama, J. Phys. B: At. Mol. Opt. Phys. 29, 1861 (1996).

[13] P. A. Vetter, D. M. Meekhof, P. K. Majumder, S. K. Lamoreaux, and E. N. Fortson, Phys. Rev. Lett. 74, 2658 (1995)

[14] N. H. Edwards, S. J. Phipp, P. E. Baird, and S. Nakayama, Phys. Rev. Lett. 74, 2654 (1995)

[15] C. S. Wood, S. C. Bennett, D. Cho, B. P. Masterson, J. L. Roberts, C. E. Tanner, and C. E. Wieman, Science 275, 1759 (1997)

[16] J. Guéna, M. Lintz, and M. A. Bouchiat, Phys. Rev. A 71, 042108 (2005).

[17] V. A. Dzuba, J. C. Berengut, V. V. Flambaum, and B. Roberts, Phys. Rev. Lett. 109, 203003 (2012)

[18] V. V. Flambaum and I. B. Khriplovich, ZhETF 79, 1656 (1980).

[19] V. V. Flambaum and I. B. Khriplovich, JETP 52, 835 (1980).

[20] V.V. Flambaum, I.B. Khriplovich, and O.P. Sushkov, Phys. Lett. B 146(6), 367 (1984).

[21] B. Desplanques, J. F. Donoghue, and B. R. Holstein, Ann. Phys. 124, 449 (1980).

[22] V. A. Dzuba, V. V. Flambaum, and I. B. Khriplovich, Z. Phys. D 1, 243 (1986)

[23] B. A. Brown, A. Derevianko, and V. V. Flambaum, Phys. Rev. C 79, 035501 (2009).

[24] A. V. Viatkina, D. Antypas, M. G. Kozlov, D. Budker, and V. V. Flambaum, arXiv:1903.00123 [physics.atomph] (2019).

[25] V. A. Dzuba, V. V. Flambaum, and Y. V. Stadnik, Phys. Rev. Lett. 119, 223201 (2017)

[26] E. N. Fortson, Y. Pang, and L. Wilets, Phys. Rev. Lett. 65, 2857 (1990)

[27] J. Zhang, R. Collister, K. Shiells, M. Tandecki, S. Aubin, J. A. Behr, E. Gomez, A. Gorelov, G. Gwinner, L. A. Orozco, M. R. Pearson, and Y. Zhao, Hyperfine Interact. 237, 150 (2016)

[28] T. Aoki, Y. Torii, B. K. Sahoo, B. P. Das, K. Harada, T. Hayamizu, K. Sakamoto, H. Kawamura, T. Inoue, A. Uchiyama, S. Ito, R. Yoshioka, K. S. Tanaka, M. Itoh,
A. Hatakeyama, and Y. Sakemi, Appl. Phys. B 123, 120 (2017).

[29] M. Nuñez Portela, E. A. Dijck, A. Mohanty, H. Bekker, J. E. Van Den Berg, G. S. Giri, S. Hoekstra, C. J. Onderwater, S. Schlesser, R. G. Timmermans, O. O. Versolato, L. Willmann, H. W. Wilschut, and K. Jungmann, Appl. Phys. B 114, 173 (2014).

[30] J. Choi and D. S. Elliott, Phys. Rev. A 93, 023432 (2016).

[31] N. Leefer, L. Bougas, D. Antypas, and D. Budker, arXiv:0912.2133 [physics.atom-ph] (2014).

[32] A. Nuyen, D. Budker, D. DeMille, and M. Zolotorev, Phys. Rev. A 56, 3453 (1997).

[33] E. Altuntas, J. Ammon, S. B. Cahn, and D. DeMille, Phys. Rev. Lett. 120, 142501 (2018)

[34] E. Altuntas, J. Ammon, S. B. Cahn, and D. Demille, Phys. Rev. A 97, 042101 (2018).

[35] S. Porsev, Y. G. Rakhlina, and M. Kozlov, JETP Letters 61, 459 (1995).

[36] V. A. Dzuba and V. V. Flambaum, Phys. Rev. A 83, 042514 (2011)

[37] K. Tsigutkin, D. Dounas-Frazer, A. Family, J. E. Stalnaker, V. V. Yashchuk, and D. Budker, Phys. Rev. Lett. 103, 071601 (2009).

[38] K. Tsigutkin, D. Dounas-Frazer, A. Family, J. E. Stalnaker, V. V. Yashchuk, and D. Budker, Phys. Rev. A 81, 032114 (2010).

[39] D. DeMille, Phys. Rev. Lett. 74, 4165 (1995)

[40] B. P. Das, Phys. Rev. A 56, 1635 (1997)

[41] M. A. Bouchiat and C. Bouchiat, J. Phys. 36, 493 (1975).

[42] M. Bouchiat and L. Pottier, Science 234, 1203 (1986).

[43] P. S. Drell and E. D. Commins, Phys. Rev. Lett. 53, 968 (1984)

[44] C. J. Bowers, D. Budker, S. J. Freedman, G. Gwinner, J. E. Stalnaker, and D. DeMille, Phys. Rev. A 59, 3513 (1999)

[45] J. E. Stalnaker, D. Budker, S. J. Freedman, J. S. Guzman, S. M. Rochester, and V. V. Yashchuk, Phys. Rev. A 73, 043416 (2006).

[46] D. R. Dounas-Frazer, K. Tsigutkin, A. Family, and D. Budker, Phys. Rev. A 82, 062507 (2010)

[47] D. Antypas, A. Fabricant, and D. Budker, Opt. Lett. 43, 002241 (2018).

[48] D. Antypas, A. Fabricant, L. Bougas, K. Tsigutkin, and D. Budker, Hyperfine Interact. 238, 21 (2017)

[49] K. Tsigutkin, J. E. Stalnaker, D. Budker, S. J. Freedman, and V. V. Yashchuk, in Proceedings of The 3rd Workshop From Parity Violation to Hadronic Structure and more... (2006).

[50] C. S. Wood, S. C. Bennett, J. L. Roberts, D. Cho, and C. E. Wieman, Can. J. Phys. 77, 7 (1999)

[51] J. E. Stalnaker, D. Budker, D. P. DeMille, S. J. Freedman, and V. V. Yashchuk, Phys. Rev. A 66, 031403 (2002)

[52] M. Tanabashi et al., Phys. Rev. D 98, 030001 (2018).

[53] M. G. Kozlov, S. G. Porsev, and W. R. Johnson, Phys. Rev. A 64, 052107 (2001).

[54] B. R. Heckel, C. E. Cramer, T. S. Cook, E. G. Adelberger, S. Schlamminger, and U. Schmidt, Phys. Rev. Lett. 97, 021603 (2006).

[55] B. R. Heckel, E. G. Adelberger, C. E. Cramer, T. S. Cook, S. Schlamminger, and U. Schmidt, Phys. Rev. D 78, 092006 (2008).

[56] G. Vasilakis, J. M. Brown, T. W. Kornack, and M. V. Romalis, Phys. Rev. Lett. 103, 261801 (2009) 
[57] S. G. Porsev, M. G. Kozlov, and Y. G. Rakhlina, Hyperfine Interact. 127, 395 (2000).

[58] Singh. A. D. and B. P. Das, JOSA B 32, 4905 (1999).

[59] M. G. Kozlov, V. A. Dzuba, and V. V. Flambaum, Phys. Rev. A. 99, 012516 (2019).

[60] M. A. Bouchiat, A. Coblentz, J. Guéna, and L. Pottier, J. Phys. 42, 985 (1981)

\section{Appendix A: Transition rates and ratios $r_{m^{\prime}}$ for the} $m=0 \rightarrow m^{\prime}=0, \pm 1$ transitions

The amplitudes for the Stark-, PV-induced and magnetic-dipole $(M 1)$ transitions between the ${ }^{1} \mathrm{~S}_{0}, m=$ $0 \rightarrow{ }^{3} \mathrm{D}_{1}, m^{\prime}=0, \pm 1$ states are given by 38 :

$$
\begin{gathered}
A_{m^{\prime}}^{\text {Stark }}=i \beta(-1)^{m^{\prime}}(\vec{E} \times \overrightarrow{\mathcal{E}})_{-m^{\prime}}, \\
A_{m^{\prime}}^{P V}=i \zeta(-1)^{m^{\prime}} \overrightarrow{\mathcal{E}}_{-m^{\prime}}, \\
A_{m^{\prime}}^{M 1}=M 1(-1)^{m^{\prime}}(\hat{k} \times \overrightarrow{\mathcal{E}})_{-m^{\prime}},
\end{gathered}
$$

where $\hat{k}$ is the unit vector related to the optical field with electric field amplitude $\overrightarrow{\mathcal{E}}$, and $M 1$ is the magneticdipole transition moment, measured in [51: $|M 1|=$ $1.33(21) \times 10^{-4} \mu_{B}$, where $\mu_{B}$ is the Bohr magneton. The geometry of applied fields in the present experiment, however, is such that $A^{M 1}$ is nominally (i.e. in the absence of experimental imperfections) out of phase with respect to $A^{\text {Stark }}$, so that the two amplitudes do not interfere. Later in this Appendix we present the leading $\mathrm{PV}$-mimicking systematic due to residual Stark- $M 1$ interference.

A second level of suppression of the effects of the $M 1$ amplitude is due to the standing-wave nature of the optical field in the PBC 60. As discussed in [38, the M1 amplitude in the presence of a standing-wave with counter-propagating field amplitudes $\overrightarrow{\mathcal{E}}_{+}$and $\overrightarrow{\mathcal{E}}_{-}$, is given by:

$$
A_{m^{\prime}}^{M 1, P B C}=M 1(-1)^{m^{\prime}}(\kappa \hat{k} \times \overrightarrow{\mathcal{E}})_{-m^{\prime}},
$$

where $\overrightarrow{\mathcal{E}}=\overrightarrow{\mathcal{E}}_{+}+\overrightarrow{\mathcal{E}}_{-}$and $\kappa=\left(\mathcal{E}_{+}-\mathcal{E}_{-}\right) / \mathcal{E}$. The amplitude of A4 is suppressed by a factor $1 / \kappa$, relative to that induced by a traveling-wave field. The suppression is $\approx$ 300 in the present experiment.

We first consider the ideal case, in which there are no stray-fields, field misalignments, or ellipticity in the optical field polarization and $\kappa=0$, and derive expressions for the three frequency components $R_{m^{\prime}}^{[0]}, R_{m^{\prime}}^{[1]}$, and $R_{m^{\prime}}^{[2]}$ present in the excitation rate $R_{m^{\prime}}$. In this case, the electric, magnetic, and optical fields are as follows:

$$
\begin{gathered}
\vec{E}=\left(E_{d c}+E_{0} \cos \omega t\right) \hat{x}, \\
\vec{B}=B_{z} \hat{z}
\end{gathered}
$$

$$
\overrightarrow{\mathcal{E}}=\mathcal{E}(\sin \theta \hat{y}+\cos \theta \hat{z})
$$

The transition rate $R_{m^{\prime}}$ is given by:

$$
\begin{array}{r}
R_{m^{\prime}} \propto\left|A_{m^{\prime}}^{\text {Stark }}+A_{m^{\prime}}^{P V}\right|^{2} \\
=R_{m^{\prime}}^{[0]}+R_{m^{\prime}}^{[1]} \cos \omega t+R_{m^{\prime}}^{[2]} \cos 2 \omega t .
\end{array}
$$

Evaluating the amplitudes of A1, A2 and A4 in A8 yields the following harmonic amplitudes for the $0 \rightarrow 0$ transition rate:

$$
\begin{array}{r}
R_{0}^{[0]} \approx 2 \mathcal{E}^{2} \beta^{2} E_{0}^{2} \sin ^{2} \theta+4 \mathcal{E}^{2} \beta^{2} E_{d c}^{2} \sin ^{2} \theta \\
+8 \mathcal{E}^{2} \beta E_{d c} \zeta \cos \theta \sin \theta \\
R_{0}^{[1]}=8 \mathcal{E}^{2} \beta E_{0} \zeta \cos \theta \sin \theta+8 \mathcal{E}^{2} \beta^{2} E_{0} E_{d c} \sin ^{2} \theta \\
R_{0}^{[2]} \approx 2 \mathcal{E}^{2} \beta^{2} E_{0}^{2} \sin ^{2} \theta
\end{array}
$$

The amplitudes for the $0 \rightarrow \pm 1$ transitions are:

$$
\begin{gathered}
R_{ \pm 1}^{[0]} \approx \mathcal{E}^{2} \beta^{2} E_{0}^{2} \cos ^{2} \theta+2 \mathcal{E}^{2} \beta^{2} E_{d c}^{2} \cos ^{2} \theta \\
-4 \mathcal{E}^{2} \beta E_{d c} \zeta \cos \theta \sin \theta \\
R_{ \pm 1}^{[1]}=-4 \mathcal{E}^{2} \beta E_{0} \zeta \cos \theta \sin \theta+4 \mathcal{E}^{2} \beta^{2} E_{0} E_{d c} \cos ^{2} \theta \\
R_{ \pm 1}^{[2]}=\mathcal{E}^{2} \beta^{2} E_{0}^{2} \cos ^{2} \theta
\end{gathered}
$$

The terms proportional to $\zeta^{2}$ were dropped in A10 A12, $\mathrm{A} 13$. The apparatus measures the ratio of the $1^{\text {st }}$ - to the $2^{\text {nd }}$ harmonic in the transition rate. When exciting the $0 \rightarrow 0$ transition, this ratio is given by:

$$
r_{0} \equiv \frac{R_{0}^{[1]}}{R_{0}^{[2]}}=\frac{4 E_{d c}}{E_{0}}+\frac{4 \zeta}{\beta E_{0}} \cot \theta .
$$

For the $0 \rightarrow \pm 1$ transition, the corresponding ratio is:

$$
r_{ \pm 1} \equiv \frac{R_{ \pm 1}^{[1]}}{R_{ \pm 1}^{[2]}}=\frac{4 E_{d c}}{E_{0}}-\frac{4 \zeta}{\beta E_{0}} \tan \theta
$$

We now derive expressions for $r_{0}$ and $r_{ \pm 1}$ in the presence of apparatus imperfections. In this case the fields $\vec{E}, \vec{B}$ and $\overrightarrow{\mathcal{E}}$ are expressed as:

$$
\vec{E}=\left(E_{d c}+E_{0} \cos \omega t\right) \hat{x}+\left(e_{y}+e_{y}^{r} \cos \omega t\right) \hat{y}+\left(e_{z}+e_{z}^{r} \cos \omega t\right) \hat{z},
$$

$$
\vec{B}=\left(b_{x}+f_{B} b_{x}^{r}\right) \hat{x}+\left(b_{y}+f_{B} b_{y}^{r}\right) \hat{y}+\left(b_{z}+f_{B} B_{z}\right) \hat{z}
$$

$$
\overrightarrow{\mathcal{E}}=\mathcal{E}\left(\sin \theta \hat{y}+\cos \theta e^{i \phi} \hat{z}\right)
$$

We include in this analysis the contribution of the M1 amplitude (eq. A4). The various terms in A18, A19. 
A20 were introduced in section II The presence of the small $b_{x}^{\prime}=b_{x}+f_{B} b_{x}^{r}$ and $b_{y}^{\prime}=b_{y}+f_{B} b_{y}^{r}$ components, in addition to the leading field $B_{z}^{\prime}=b_{z}+f_{B} B_{z}$, is responsible for Zeeman mixing of adjacent $m^{\prime}$ sublevels of the ${ }^{3} \mathrm{D}_{1}$ state, which needs to be considered in deriving the expressions for the transition rate $R_{m^{\prime}}$ and the harmonics ratios $r_{m^{\prime}}$. One approach is to compute this mixing and modify the amplitudes of A1, A2 and A3 accordingly. Alternatively, the fields $\vec{E}, \vec{B}$ and $\overrightarrow{\mathcal{E}}$ can be rotated by application of an operator $\mathcal{D}=D\left(-a_{y}, \hat{y}\right) D\left(a_{x}, \hat{x}\right)$, such that $\mathcal{D} \vec{B} \propto \hat{z}\left[38\right.$. The rotation angles are $a_{x(y)}=$ $b_{y(x)}^{\prime} / B_{z}^{\prime}$. The rotated fields $\mathcal{D} \vec{E}, \mathcal{D} \vec{B}, \mathcal{D} \hat{k}$ and $\mathcal{D} \overrightarrow{\mathcal{E}}$ are used to evaluate the transition amplitudes of eqns $\mathrm{A} 1$. A2 and A3). A great number of terms appear then in the expression for the rates $R_{m^{\prime}}$. The corresponding harmonics ratios $r_{m^{\prime}}$ are expanded in terms of the small field imperfections, the parameter $\kappa$ and $\zeta$. The ratios $r_{0}$ and $r_{ \pm 1}$, retaining terms up to $2^{\text {nd }}$ order in the expansion, are as follows:

$$
\begin{gathered}
r_{0}\left(\theta, f_{B}\right)=\frac{4 E_{d c}}{E_{0}}-\frac{4\left(b_{x}+f_{B} b_{x}^{r}\right) e_{z}}{f_{B} B_{z} E_{0}}+ \\
{\left[\frac{4 \zeta}{\beta E_{0}}+\frac{4\left(b_{x}+f_{B} b_{x}^{r}\right) e_{y}}{f_{B} B_{z} E_{0}}\right] \cot \theta \cos \phi} \\
\quad+\frac{4\left(b_{y}+f_{B} b_{y}^{r}\right) \zeta}{f_{B} B_{z} E_{0}}\left(1+\cot ^{2} \theta \cos 2 \phi\right), \quad(\mathrm{A} 21) \\
r_{ \pm 1}\left(\theta, f_{B}\right)=\frac{4 E_{d c}}{E_{0}}+\frac{4 e_{y}^{r} e_{y}}{E_{0}^{2}} \pm \frac{4 e_{y}^{r} M 1 \kappa}{\beta E_{0}^{2}}+\frac{4\left(b_{y}+f_{B} b_{y}^{r}\right) \zeta}{f_{B} B_{z} \beta E_{0}}+ \\
{\left[-\frac{4 \zeta}{\beta E_{0}}-\frac{4\left(b_{x}+f_{B} b_{x}^{r}\right) e_{y}}{f_{B} B_{z} E_{0}}-\frac{4 e_{y}^{r} e_{z}}{E_{0}^{2}}-\frac{4 e_{z}^{r} e_{y}}{E_{0}^{2}} \mp \frac{4 e_{z}^{r} M 1 \kappa}{\beta E_{0}^{2}}\right]} \\
\quad \times \tan \theta \cos \phi \pm \frac{4 e_{z}}{E_{0}} \tan \theta \sin \phi+ \\
{\left[\frac{4\left(b_{x}+f_{B} b_{x}^{r}\right) e_{z}}{f_{B} B_{z} E_{0}}+\frac{4 e_{z}^{r} e_{z}}{E_{0}^{2}}+\frac{4 b_{y} \zeta}{f_{B} B_{z} E_{0}}\right] \tan ^{2} \theta \cos 2 \phi}
\end{gathered}
$$

To compare the sensitivity of $\zeta / \beta$ measurements, made in different transition components, to false-PV effects, we form the combination $K_{1}$ [see eq. (13) and Table I] with use of $r_{0}$ and the sum $(1 / 2)\left(r_{-1}+r_{+1}\right)$ :

$$
K_{1}^{0}=\left[\frac{8 \zeta}{\beta E_{0}}+\frac{8 b_{x}^{r} e_{y}}{B_{z} E_{0}}\right]\left(\cot \theta_{+} \cos \phi_{+}-\cot \theta_{-} \cos \phi_{-}\right)
$$

$$
\begin{array}{r}
K_{1}^{ \pm 1}=\left[-\frac{8 \zeta}{\beta E_{0}}-\frac{8 b_{x}^{r} e_{y}}{B_{z} E_{0}}-\frac{8 e_{y}^{r} e_{z}}{E_{0}^{2}}-\frac{8 e_{z}^{r} e_{y}}{E_{0}^{2}}\right] \times \\
\left(\tan \theta_{+} \cos \phi_{+}-\tan \theta_{-} \cos \phi_{-}\right) .
\end{array}
$$

We see that there are more false-PV terms in $K_{1}^{ \pm 1}$, compared to $K_{1}^{0}$. A misalignment such that $e_{y}^{r} / E_{0}=0.005$, for instance, coupling to a stray $e_{z}=50 \mathrm{mV} / \mathrm{cm}$, gives rise to a PV-mimicking signal of $0.25 \mathrm{mV} / \mathrm{cm}$, which is $\approx 1 \%$ of the measured PV effect. This is the primary reason why the isotopic comparison data were taken at the $0 \rightarrow 0$ transition component.

We now evaluate the impact of the $M 1$-related PVmimicking contributions, to separately illustrate the effectiveness of the two methods used to suppress the effects of the magnetic dipole transition, namely the choice of experimental field geometry and the excitation of the $408 \mathrm{~nm}$ transition with a standing-wave field. We focus on the $m=0 \rightarrow m^{\prime}=0$ component of the ${ }^{1} \mathrm{~S}_{0} \rightarrow{ }^{3} \mathrm{D}_{1}$ transition, however, analysis on the $m=0 \rightarrow m^{\prime}= \pm 1$ components yields similar conclusions. We start by evaluating the suppression provided by the experimental field geometry. For this, we compute the harmonics ratio $r_{0}\left(\theta, f_{B}\right)$ and the associated combination $K_{1}^{0}$, assuming that a traveling-wave field excites atoms, i.e. the parameter $\kappa$ of $\mathrm{A} 4$ is not negligible. If the ratio $r_{0}\left(\theta, f_{B}\right)$ is expanded in terms of the various field imperfections, a third order term in the small parameters that contains the $M 1$ amplitude appears in $r_{0}\left(\theta, f_{B}\right)$, which mimics the PV-term. We omit the number of contributions from Stark-induced systematics, and focus on the competition between the M1- and PV-related signals. The relevant part of the combination $K_{1}^{0}$ is:

$$
\begin{aligned}
& K_{1}^{0}=\left(\frac{8 \zeta}{\beta E_{0}}\right)\left(\cot \theta_{+} \cos \phi_{+}-\cot \theta_{-} \cos \phi_{-}\right) \\
& -\left(\frac{8 b_{x}^{r} e_{y}^{r} \kappa M 1}{B_{z} \beta E_{0}^{2}}\right)\left(\cot \theta_{+} \sin \phi_{+}-\cot \theta_{-} \sin \phi_{-}\right) .
\end{aligned}
$$

Let us form the ratio $r_{M 1-P V}$ of the $M 1$ - and PV-related contributions to $K_{1}^{0}$ :

$$
\begin{aligned}
r_{M 1-P V}=- & \left(\frac{M 1 / \beta}{\zeta / \beta}\right)\left(\frac{b_{x}^{r} e_{y}^{r}}{B_{z} E_{0}}\right) \kappa \\
& \times \frac{\cot \theta_{+} \sin \phi_{+}-\cot \theta_{-} \sin \phi_{-}}{\cot \theta_{+} \cos \phi_{+}-\cot \theta_{-} \cos \phi_{-}} .
\end{aligned}
$$

The ratio $M 1 / \beta \approx-22.3 \mathrm{~V} / \mathrm{cm}$ [51], $\zeta / \beta \approx-23.9$ $\mathrm{mV} / \mathrm{cm}$, and $\cot \theta_{ \pm} \approx \pm 1$. Assuming reasonable values for imperfections: $b_{x}^{r} / B_{z}=10^{-4}, e_{y}^{r} / E_{0}=0.005$, $\phi_{ \pm}=0.05 \mathrm{rad}$, we obtain $r_{M 1-P V}=-2.3 \cdot 10^{-5} \kappa$. Therefore, the present choice of experimental field geometry is sufficient to provide a practically complete suppression of the contribution of the M1-related systematic, even if the experiment were to be carried out with a travelling-wave field to excite atoms $(\kappa=1)$. The standing-wave field in the PBC provides further suppression $(\kappa \approx 1 / 300)$, resulting in a residual fractional contribution of the PVmimicking signal due to the $M 1$ amplitude of $\approx 7.8 \cdot 10^{-8}$.

In section IV A 2 we discussed the measurement of the polarization ellipticity-related angle $\phi$. This angle is determined by combining measurements of the difference $r_{+1}-r_{-1}$, made for opposite polarities of an enhanced 
$e_{z}$ field $\left( \pm e_{z}\right)$. Use of $\mathrm{A} 22$ in this case yields:

$$
\begin{aligned}
&\left(r_{+1}-r_{-1}\right)_{+e_{z}}-\left(r_{+1}-r_{-1}\right)_{-} e_{z}= \\
& \frac{16 e_{z}}{E_{0}} \tan \theta \sin \phi
\end{aligned}
$$

This expression was used in the analysis presented in section IV A 2 .

\section{Appendix B: Measuring $\theta_{ \pm}$using the $408 \mathrm{~nm}$ profile}

Here we describe the method to measure the polarization angles $\theta_{ \pm}$using recorded profiles of the $408 \mathrm{~nm}$ resonance. These measurements are correlated with the concurrent readings of a polarimeter monitoring the light transmitted through the $\mathrm{PBC}$, whose subsequent readings during a PV run are used to provide continuous tracking of the $\theta_{ \pm}$angles.

The polarization angles input to the $\mathrm{PBC}$ are set to approximately $\pm \pi / 4$ (i.e. to the nominal values for which PV data are acquired) and are determined through analysis of the relative peak heights for the three transition components $m=0 \rightarrow m^{\prime}=0, \pm 1$. Let $R_{0}^{[2]^{\prime}}, R_{ \pm 1}^{[2]^{\prime}}$ be the $2^{\text {nd }}$ harmonic amplitudes of the $0 \rightarrow 0$ and $0 \rightarrow \pm 1$ transitions, given by:

$$
\begin{gathered}
R_{0}^{[2]^{\prime}}=R_{0}^{[2]}+h\left(R_{-1}^{[2]}+R_{+1}^{[2]}\right) \\
R_{ \pm 1}^{[2]^{\prime}}=R_{ \pm 1}^{[2]}+h R_{0}^{[2]}
\end{gathered}
$$

where $R_{m^{\prime}}^{[2]}$ is the amplitude of the $m^{\prime}$ transition component in the absence of peak overlap, and $h=0.00042(4)$ [introduced in (21)] is a parameter quantifying the slight overlap of adjacent peaks in the spectrum. The amplitudes $R_{m^{\prime}}^{[2]}$ include a small correction for the slight saturation of the corresponding transitions (see section IV A 1.)
We form the quantity:

$$
L\left(\theta, f_{B} ; x_{i}\right)=\frac{1}{2} \frac{R_{0}^{[2]^{\prime}}-R_{-1}^{[2]^{\prime}}-R_{+1}^{[2]^{\prime}}}{R_{0}^{[2]^{\prime}}+R_{-1}^{[2]^{\prime}}+R_{+1}^{[2]^{\prime}}} .
$$

This parameter is a function of $\theta$, the magnetic field flipping parameter $f_{B}$, and all apparatus imperfections (i.e. field imperfections and $h$ ), which we label as $x_{i}$. When $x_{i} \rightarrow 0$ then $L\left(\theta, f_{B}\right)=-(1 / 2) \cos 2 \theta$, and $L=0$ for $\theta= \pm \pi / 4$. We adjust the input to the $\mathrm{PBC}$ polarization angles for an $L \approx 0$ reading (to within $1 \cdot 10^{-3}$ ), and use the measured values of $L$ to determine the actual $\theta_{+}$and $\theta_{-}$angles. For a given angle, we average measurements made for both polarities of the magnetic field $\left(f_{B}= \pm 1\right)$ :

$$
\bar{L}_{ \pm}=\frac{1}{2}\left[L\left(\theta_{ \pm}, f_{B}=+1 ; x_{i}\right)+L\left(\theta_{ \pm}, f_{B}=-1 ; x_{i}\right)\right] .
$$

We use an approximate formula to relate $\bar{L}_{ \pm}$to $\theta_{ \pm}$, that is derived by series expansion of (B4) in the small parameters $x_{i}$, and in $\theta_{ \pm}$around $\pm \pi / 4$, respectively:

$$
\bar{L}_{ \pm} \approx\left( \pm \theta_{ \pm}-\frac{\pi}{4}\right) \mp \frac{b_{y}^{r}}{B_{z}}-\frac{h}{4} .
$$

The $\theta_{ \pm}$angles corresponding to measured $\bar{L}_{ \pm}$values are given by:

$$
\theta_{ \pm} \approx \pm \frac{\pi}{4} \pm \bar{L}_{ \pm}+\frac{b_{y}^{r}}{B_{z}} \pm \frac{h}{4}
$$

We see from $\mathrm{B} 6$ that $\theta_{ \pm}$can only be determined with an offset $b_{y}^{r} / B_{z}$ (estimated to be as large as a few parts per $10^{3}$ ), which we do not have an accurate way to measure in the current apparatus [we do make a correction to $\theta_{ \pm}$to account for the contribution of the parameter $h$ present in (B6)]. This offset, however, does not affect the determination of the parameter $p_{\theta}(18)$, used to calibrate the PV data. To show this, we expand $p_{\theta}=\cot \theta_{+}-$ $\cot \theta_{-}$around $\theta_{+}=\pi / 4$ and $\theta_{-}=-\pi / 4$ :

$$
p_{\theta} \approx 2\left(1+\frac{\pi}{2}-\theta_{+}+\theta_{-}\right),
$$

or, with use of $\mathrm{B} 6$ :

$$
p_{\theta} \approx 2\left(1-\bar{L}_{+}-\bar{L}_{-}-\frac{h}{2}\right) \text {. }
$$

We see that $p_{\theta}$ is independent of the imperfection $b_{y}^{r} / B_{z}$. 